Supporting Information for

\title{
Enamines from Terminal Epoxides and Hindered Lithium Amides
}

David M. Hodgson, ${ }^{\dagger}{ }^{\dagger}$ Christopher D. Bray ${ }^{\dagger}$ and Nicholas D. Kindon ${ }^{\dagger}$

'Department of Chemistry, University of Oxford, Chemistry Research Laboratory, Mansfield Road, Oxford OXI 3QY, U.K.

${ }^{*}$ AstraZeneca, Medicinal Chemistry R\&D Charnwood, Bakewell Road, Loughborough, Leicestershire LE11 5RH, U.K.

\section{General Details}

All reactions requiring anhydrous conditions were conducted in flame-dried apparatus under an atmosphere of argon. THF was distilled from benzophenone ketyl; TMP, benzene- $\mathrm{d}_{6}$ and acetonitrile- $\mathrm{d}_{3}$ from $\mathrm{CaH}_{2}$; all other reagents were used as received unless stated otherwise. Column chromatography was carried out on Kieselgel $60(40-63 \mu \mathrm{m})$. Silica was deactivated by stirring with neat $\mathrm{NEt}_{3}$ for $12 \mathrm{~h}$. Light petrol refers to the fraction of petroleum boiling between $30{ }^{\circ} \mathrm{C}$ and $40{ }^{\circ} \mathrm{C}$. IR spectra were recorded as thin films, using a Perkin-Elmer 1750 FTIR spectrophotometer. Peak intensities are specified as strong (s), medium (m) or weak (w). ${ }^{1} \mathrm{H}$ and ${ }^{13} \mathrm{C}$ NMR spectra were recorded in $\mathrm{CDCl}_{3}$ at 25 ${ }^{\circ} \mathrm{C}$, with Bruker DPX250, DPX400, DQX400 or AMX500 spectrometers unless otherwise stated. Chemical shifts are reported relative to $\mathrm{CDCl}_{3}\left({ }^{1} \mathrm{H}\right.$ NMR $7.27 ;{ }^{13} \mathrm{C}$ NMR 77.0 (central signal of triplet)) unless stated otherwise. Coupling constants $(J)$ are given in $\mathrm{Hz}$, multiplicities are given as multiplet $(\mathrm{m})$, singlet (s) (and where applicable are described as broad (b)), doublet (d), triplet (t), quartet (q) and septet (sept). Mass spectra were obtained using a Micromass GCT spectrometer or by the EPSRC National Mass Spectrometry Service Centre at the University of Swansea using a 900 XLT high resolution double focusing mass spectrometer with tandem Ion Trap.

Epoxides in Table 1 were either commercially available (1,2-Epoxyoctadecane (tech.) was recrystallized and 1,2-epoxypentane, 3-methyl-1,2-epoxybutane, 1,2-epoxy-9-decene, 1,2-epoxyhexane and 1,2,7,8-diepoxyoctane were distilled prior to use) or prepared according to: (a) Elings, J. A.; Downing, R. S.; Sheldon, R. A. Eur. J. Org. Chem. 1999, 837-846 (entry 4); (b) Yang, L.; Weber, A. E.; Greenlee, W. J.; Patchett, A. A. Tetrahedron Lett. 1993, 34, 7035-7038 (entry 5); (c) Hodgson, D. M.; Reynolds, N. J.; Coote, S. J. Tetrahedron Lett. 2002, 43, 7895-7897 (entry 6); (d) 2,5,5-Trimethyl2-(2-oxiranyl-ethyl)-[1.3]dioxane (entry 7) was synthesized from 5-hexen-2-one by a two-step 
sequence: (i) $\mathrm{Me}_{2} \mathrm{C}\left(\mathrm{CH}_{2} \mathrm{OH}\right)_{2}$ (1.3 equiv.), benzene, $\Delta, 4 \mathrm{~h}$, azeotrope (76\%); (ii) $m$-CPBA (1.5 equiv.), $\mathrm{CH}_{2} \mathrm{Cl}_{2}, \mathrm{KHCO}_{3}\left(0.5 \mathrm{M}\right.$ in $\left.\mathrm{H}_{2} \mathrm{O}\right), 16 \mathrm{~h}(38 \%)$; IR $\left(\mathrm{cm}^{-1}\right) 2982 \mathrm{~m}, 2955 \mathrm{~s}, 2968 \mathrm{~s}, 1471 \mathrm{~m}, 1457 \mathrm{~m}, 1396 \mathrm{~m}$, $1373 \mathrm{~m}, 1276 \mathrm{~m}, 1250 \mathrm{~m}, 1212 \mathrm{~m}, 1191 \mathrm{w}, 1169 \mathrm{w}, 1123 \mathrm{~m}, 1093 \mathrm{~s}, 1040 \mathrm{~m}, 1021 \mathrm{~m} ;{ }^{1} \mathrm{H}$ NMR $(400 \mathrm{MHz})$ 3.54-3.37 (m, 4H, $\left.2 \times \mathrm{OCH}_{2}\right), 2.95-2.90(\mathrm{~m}, 1 \mathrm{H}, \mathrm{OCH}), 2.71(\mathrm{dd}, 1 \mathrm{H}, J=5$ and 5, OCH), $2.45(\mathrm{dd}, 1 \mathrm{H}$, $J=5$ and 3, OCH), 1.88-1.56 (m, 4H, $\left.\mathrm{CH}_{2} \mathrm{CH}_{2}\right), 1.33$ (s, 3H, Me), 0.98 (s, 3H, CMeMe), $0.84(\mathrm{~s}, 3 \mathrm{H}$, $\mathrm{CMeMe}) ;{ }^{13} \mathrm{C} \mathrm{NMR}(100 \mathrm{MHz}) 98.4\left(\mathrm{O}_{2} \mathrm{C}\right), 70.3(2 \times \mathrm{OC}), 52.2\left(\mathrm{OCH}_{2}\right), 47.1(\mathrm{OCH}), 34.3\left(\mathrm{CH}_{2}\right), 29.8$ $\left(\mathrm{CMe}_{2}\right), 26.5\left(\mathrm{CH}_{2}\right), 22.7(\mathrm{CMeMe}), 22.4(\mathrm{CMeMe}), 20.1(\mathrm{Me})$; HRMS m/z $\left(\mathrm{M}+\mathrm{H}^{+}\right)$Found: 201.1493. $\mathrm{C}_{11} \mathrm{H}_{21} \mathrm{O}_{3}$ requires 201.1490; (e) Rothberg, I.; Schneider, L.; Kirsch, S.; OFee, R. J. Org. Chem. 1982, 47, 2675-2676 (entry 8). $N$-(2-hydroxyoctadecyl)-TMP was prepared by ring opening of 1,2epoxyoctadecane with TMP ( 3 equiv. $\mathrm{K}_{2} \mathrm{CO}_{3}, i-\mathrm{PrOH}, 100{ }^{\circ} \mathrm{C}$ (sealed tube), $16 \mathrm{~h}$ ) in $6 \%$ yield. $N$-tertButylpinacoylamine ${ }^{1}$ was prepared from pinacolone and tert-butylamine using the method described by Stowell and Padegimas in 34\% yield.

\section{General procedure for the formation of enamines from terminal epoxides and LTMP:}

\section{2,2,6,6-Tetramethyl-1-octadec-1-enylpiperidine 2a}

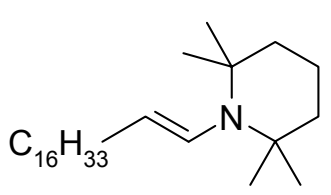

To a solution of 2,2,6,6-tetramethylpiperidine $\left(0.50 \mathrm{~cm}^{3}, 2.96 \mathrm{mmol}\right)$ in THF (6 $\left.\mathrm{cm}^{3}\right)$ at $0{ }^{\circ} \mathrm{C}$ was added $n$-BuLi $\left(1.6 \mathrm{~mol} \mathrm{dm}{ }^{-3}\right.$ in hexanes; $\left.1.85 \mathrm{~cm}^{3}, 2.96 \mathrm{mmol}\right)$ dropwise. The solution was allowed to warm to $25{ }^{\circ} \mathrm{C}$ over $15 \mathrm{~min}$. before a solution of 1,2-epoxyoctadecane (318 $\mathrm{mg}, 1.19 \mathrm{mmol})$ in THF $\left(1.5 \mathrm{~cm}^{3}\right)$ was added in one portion. The reaction mixture was stirred at $25^{\circ} \mathrm{C}$ for $1 \mathrm{~h}$ before being filtered through a pad of deactivated silica $(5$ $\mathrm{cm} \times 5 \mathrm{~cm})$. The pad was washed with $5 \% \mathrm{NEt}_{3}$ in light petrol $\left(250 \mathrm{~cm}^{3}\right)$. The solvent and amines were removed in vacuo (down to $0.1 \mathrm{mbar}, 50{ }^{\circ} \mathrm{C}$ ) to give enamine 2a $(361 \mathrm{mg}, 78 \%$ ) as a glassy solid; IR $\left(\mathrm{cm}^{-1}\right)$ 2924s, 2853s, 1641w (C=C), 1465m, 1376w, 1382w, 1265w, 1246w, 1174w, 1131w, 1080w, 1033w; ${ }^{1} \mathrm{H}$ NMR (500 MHz, $\left.\mathrm{C}_{6} \mathrm{D}_{6}\right) 5.79(\mathrm{~d}, 1 \mathrm{H}, J=14, \mathrm{NCH}), 5.32(\mathrm{dt}, 1 \mathrm{H}, J=14$ and $7,=\mathrm{CH}), 2.08$ $\left(\mathrm{dt}, 2 \mathrm{H}, J=7\right.$ and $\left.7,=\mathrm{CHCH}_{2}\right), 1.51-1.18\left(\mathrm{~m}, 34 \mathrm{H}, 17 \times \mathrm{CH}_{2}\right), 1.13\left(\mathrm{~s}, 12 \mathrm{H}, 2 \times \mathrm{NCMe}_{2}\right), 0.91(\mathrm{t}, 3 \mathrm{H}, J$ $=7, \mathrm{Me}) ;{ }^{13} \mathrm{C}$ NMR $\left(125 \mathrm{MHz}, \mathrm{C}_{6} \mathrm{D}_{6}\right) 131.8(\mathrm{NCH}=), 126.2(=\mathrm{CH}), 53.7\left(2 \times \mathrm{CMe}_{2}\right), 41.6(2 \times$ 
$\left.\mathrm{CMe}_{2} \mathrm{CH}_{2}\right), 32.4\left(=\mathrm{CHCH}_{2}\right), 32.1\left(\mathrm{CH}_{2}\right), 31.1\left(\mathrm{CH}_{2}\right), 30.9\left(\mathrm{CH}_{2}\right), 30.2-29.7\left(12 \times \mathrm{CH}_{2}\right) \quad 28.0(2 \times$ $\left.\mathrm{NCMe}_{2}\right), 23.1\left(\mathrm{CH}_{2}\right), 18.1\left(\mathrm{CH}_{2}\right), 14.4(\mathrm{Me})$. HRMS m/z $\left(\mathrm{M}+\mathrm{H}^{+}\right)$Found: $392.4254 . \mathrm{C}_{27} \mathrm{H}_{54} \mathrm{~N}$ requires 392.4251.

2,2,6,6-Tetramethyl-1-(pent-1-enyl)-piperidine $\mathbf{2 b}$

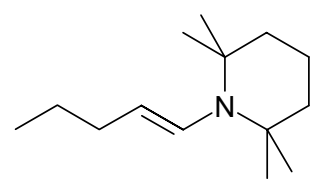

According to the general procedure, 1,2-epoxypentane (204 $\mathrm{mg}, 2.37 \mathrm{mmol})$ gave, following bulb-to-bulb distillation, enamine $\mathbf{2 b}(371 \mathrm{mg}, 75 \%)$ as a colorless oil; bp $60{ }^{\circ} \mathrm{C} / 0.06$ mbar; IR $\left(\mathrm{cm}^{-1}\right) 2965 \mathrm{~s}, 2928 \mathrm{~s}, 2871 \mathrm{~m}, 1662 \mathrm{~m}(\mathrm{C}=\mathrm{C}), 1641 \mathrm{~m}$ $(\mathrm{C}=\mathrm{C}), 1463 \mathrm{~m}, 1376 \mathrm{~m}, 1362 \mathrm{~m}, 1353 \mathrm{~m}, 1265 \mathrm{~m}, 1246 \mathrm{w}, 1174 \mathrm{w}, 1147 \mathrm{w}, 1131 \mathrm{~m}, 1031 \mathrm{w}, 956 \mathrm{~s} ;{ }^{1} \mathrm{H}$ NMR $\left(400 \mathrm{MHz}, \mathrm{C}_{6} \mathrm{D}_{6}\right) 5.78(\mathrm{dt}, 1 \mathrm{H}, J=14$ and 1, NCH), $5.30(\mathrm{dt}, 1 \mathrm{H}, J=14$ and 7, =CH), $2.01(\mathrm{dt}, 2 \mathrm{H}, J=$ 7 and 7, $\left.=\mathrm{CHCH}_{2}\right), 1.47-1.43\left(\mathrm{~m}, 6 \mathrm{H}, 3 \times \operatorname{ring} \mathrm{CH}_{2}\right), 1.38\left(\mathrm{tq}, 2 \mathrm{H}, J=7\right.$ and $\left.7, \mathrm{CH}_{2} \mathrm{Me}\right), 1.13(\mathrm{~s}, 12 \mathrm{H}, 2$ $\left.\times \mathrm{NCMe}_{2}\right), 0.91\left(\mathrm{t}, 3 \mathrm{H}, J=7, \mathrm{CH}_{2} \mathrm{Me}\right) ;{ }^{13} \mathrm{C} \mathrm{NMR}\left(100 \mathrm{MHz}, \mathrm{C}_{6} \mathrm{D}_{6}\right) 132.2(\mathrm{NCH}=), 126.0(=\mathrm{CH}), 53.9$ $\left(2 \times \mathrm{CMe}_{2}\right), 41.8\left(2 \times \mathrm{CMe}_{2} \mathrm{CH}_{2}\right), 33.4\left(=\mathrm{CHCH}_{2}\right), 28.2\left(2 \times \mathrm{NCMe}_{2}\right), 24.2\left(\mathrm{CH}_{2}\right), 18.3\left(\mathrm{CH}_{2}\right), 14.2$ (Me). HRMS $m / z\left(\mathrm{M}+\mathrm{H}^{+}\right)$Found: 210.2221. $\mathrm{C}_{14} \mathrm{H}_{28} \mathrm{~N}$ requires 210.2222.

Alternatively, to a solution of $N$-formyl-TMP $(430 \mathrm{mg}, 2.54 \mathrm{mmol})$ in $\mathrm{Et}_{2} \mathrm{O}\left(1 \mathrm{~cm}^{3}\right)$ at $-15^{\circ} \mathrm{C}$ was added $n$-BuMgCl (2.0 mol dm${ }^{-3}$ in THF; $\left.1.00 \mathrm{~cm}^{3}, 2.00 \mathrm{mmol}\right)$ dropwise. The solution was stirred at room temperature for 7 days. The solvent was then removed in vacuo, dry $n$-pentane $\left(2 \times 2.5 \mathrm{~cm}^{3}\right)$ was added and the supernatant liquid filtered (syringe filter) to give a clear solution. The solvent was removed in vacuo and the residue was purified by bulb-to-bulb distillation to give enamine $\mathbf{2 b}(133 \mathrm{mg}, 32 \%)$ as colorless oil. Data as above.

\section{2,2,6,6-Tetramethyl-1-(3-methyl-but-1-enyl)-piperidine 2c}

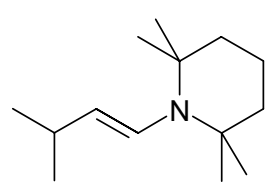

According to the general procedure, 3-methyl-1,2-epoxybutane (204 mg, $2.37 \mathrm{mmol})$ gave, following removal of the solvent from the reaction mixture under a stream of argon and direct distillation of the residue, enamine 2c (297 $\mathrm{mg}, 60 \%)$ as a colorless 
oil; bp $60{ }^{\circ} \mathrm{C} / 0.06$ mbar; IR $\left(\mathrm{cm}^{-1}\right) 2964 \mathrm{~s}, 2930 \mathrm{~s}, 2869 \mathrm{~s}, 1662 \mathrm{w}(\mathrm{C}=\mathrm{C}), 1641 \mathrm{~m}(\mathrm{C}=\mathrm{C}), 1544 \mathrm{w}, 1465 \mathrm{~m}$, $1376 \mathrm{~m}, 1362 \mathrm{~m}, 1266 \mathrm{~m}, 1246 \mathrm{~m}, 1202 \mathrm{w}, 1174 \mathrm{~m}, 1131 \mathrm{~m}, 1066 \mathrm{w}, 1033 \mathrm{~m}, 960 \mathrm{~m}, 919 \mathrm{w}, 874 \mathrm{w}, 838 \mathrm{w}$, 800w, 694w; ${ }^{1} \mathrm{H}$ NMR (400 MHz, $\left.\mathrm{C}_{6} \mathrm{D}_{6}\right)$ 5.74(dd, $1 \mathrm{H}, J=14$ and $\left.1, \mathrm{NCH}\right), 5.26(\mathrm{dd}, 1 \mathrm{H}, J=14$ and 7 , $=\mathrm{CH}), 2.30(\mathrm{dsept}, 1 \mathrm{H}, J=14$ and $7, \mathrm{CH}), 1.50-1.35\left(\mathrm{~m}, 6 \mathrm{H}, 3 \times \operatorname{ring} \mathrm{CH}_{2}\right), 1.11\left(\mathrm{~s}, 12 \mathrm{H}, 2 \times \mathrm{NCMe}_{2}\right)$, $1.02(\mathrm{~d}, 6 \mathrm{H}, J=7, \mathrm{CHMe}) ;{ }^{13} \mathrm{C} \mathrm{NMR}\left(100 \mathrm{MHz}, \mathrm{C}_{6} \mathrm{D}_{6}\right) 134.0(\mathrm{NCH}=), 129.5(=\mathrm{CH}), 53.9\left(2 \times C \mathrm{Me}_{2}\right)$, $41.8\left(2 \times \mathrm{CMe}_{2} \mathrm{CH}_{2}\right), 30.7(=\mathrm{CHC}), 28.2\left(2 \times \mathrm{NCMe}_{2}\right), 24.0(\mathrm{CHMe} 2), 18.3\left(\mathrm{CH}_{2}\right) ; \mathrm{HRMS} m / z\left(\mathrm{M}+\mathrm{H}^{+}\right)$ Found: $210.2220 . \mathrm{C}_{14} \mathrm{H}_{28} \mathrm{~N}$ requires 210.2222.

1-Deca-1,9-dienyl-2,2,6,6-tetramethylpiperidine 2d

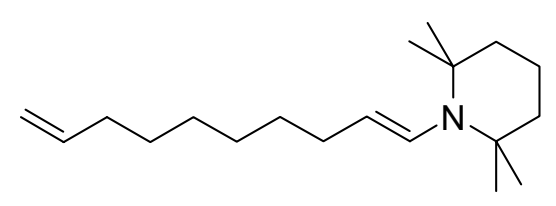

According to the general procedure, 1,2-epoxy-9-decene (154 mg, $1.00 \mathrm{mmol}$ ) gave enamine $\mathbf{2 d}$ as a colorless oil (230 $\mathrm{mg}, 83 \%)$; IR $\left(\mathrm{cm}^{-1}\right) 3076 \mathrm{w}, 2966 \mathrm{~s}, 2927 \mathrm{~s}, 2854 \mathrm{~s}, 1661 \mathrm{~m}(\mathrm{C}=\mathrm{C}), 1641 \mathrm{~m}(\mathrm{C}=\mathrm{C})$,

$1463 \mathrm{~m}, 1376 \mathrm{~m}, 1362 \mathrm{~m}, 1266 \mathrm{~m}, 1246 \mathrm{~m}, 1201 \mathrm{w}, 1174 \mathrm{~m}, 1131 \mathrm{~m}, 1080 \mathrm{w}, 1033 \mathrm{w} ;{ }^{1} \mathrm{H}$ NMR $(500 \mathrm{MHz}$, $\left.\mathrm{C}_{6} \mathrm{D}_{6}\right)$ 5.79-5.73 (m, 2H, $\mathrm{CH}_{2}=\mathrm{CH}$ and $\left.\mathrm{NCH}\right), 5.28(\mathrm{dt}, 1 \mathrm{H}, J=14$ and $7, \mathrm{NCH}=\mathrm{CH}), 5.03-4.95(\mathrm{~m}, 2 \mathrm{H}$, $\left.=\mathrm{CH}_{2}\right), 2.04-1.97\left(\mathrm{~m}, 4 \mathrm{H}, 2 \times=\mathrm{CHCH}_{2}\right), 1.46-1.26\left(\mathrm{~m}, 14 \mathrm{H}, 7 \times \mathrm{CH}_{2}\right), 1.11\left(\mathrm{~s}, 12 \mathrm{H}, 2 \times \mathrm{CMe}_{2}\right) ;{ }^{13} \mathrm{C}$ NMR (125 MHz, $\left.\mathrm{C}_{6} \mathrm{D}_{6}\right) 139.6\left(=\mathrm{CH}_{2}\right), 132.1(\mathrm{NCH}), 126.5(\mathrm{NCH}=\mathrm{CH}), 114.9\left(\mathrm{CH}=\mathrm{CH}_{2}\right), 54.1(2$ $\left.\times C \mathrm{Me}_{2}\right), 41.9\left(2 \times \mathrm{CMe}_{2} \mathrm{CH}_{2}\right), 34.6\left(\mathrm{CH}_{2}\right), 31.4\left(\mathrm{CH}_{2}\right), 31.2\left(\mathrm{CH}_{2}\right), 29.9\left(\mathrm{CH}_{2}\right), 29.7\left(2 \times \mathrm{CH}_{2}\right), 28.4(2 \times$ $\mathrm{CMe}_{2}$ ), 18.2 (ring $\left.\mathrm{CH}_{2}\right)$; HRMS $m / z\left(\mathrm{M}+\mathrm{H}^{+}\right)$Found: 278.2848. $\mathrm{C}_{19} \mathrm{H}_{36} \mathrm{~N}$ requires 278.2848.

2,2,6,6-Tetramethyl-1-(4-phenyl-1-but-1-enyl)-piperidine $\mathbf{2 e}$

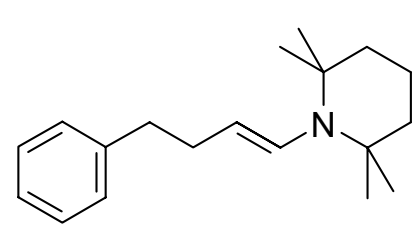

According to the general procedure, 1,2-epoxy-4-phenylbutane $(351 \mathrm{mg}$, $2.37 \mathrm{mmol})$ gave enamine $\mathbf{2 e}$ as a colorless oil (463 $\mathrm{mg}, 72 \%)$; IR $\left(\mathrm{cm}^{-1}\right)$ 3027m, 2966s, 2928s, 2869m, 1660w (C=C), 1641m (C=C), 1604w, 1496m, $1453 \mathrm{~m}, 1376 \mathrm{~m}, 1382 \mathrm{~m}, 1266 \mathrm{~m}, 1246 \mathrm{~m}, 1200 \mathrm{w}, 1174 \mathrm{~m}, 1131 \mathrm{~m}, 1030 \mathrm{w}, 955 \mathrm{~m} ;{ }^{1} \mathrm{H}$ NMR (400 MHz, $\left.\mathrm{C}_{6} \mathrm{D}_{6}\right)$ 7.24-7.05 (m, 5H, $\left.5 \times \mathrm{ArH}\right), 5.74(\mathrm{dt}, 1 \mathrm{H}, J=14$ and $1, \mathrm{NCH}), 5.18(\mathrm{dt}, 1 \mathrm{H}, J=14$ and 7, $=\mathrm{CH})$, 2.67-2.58 (m, 2H, $\left.\mathrm{PhCH}_{2}\right), 2.32\left(\mathrm{dt}, 2 \mathrm{H}, J=7\right.$ and 1, $\left.=\mathrm{CHCH}_{2}\right), 1.49-1.38\left(\mathrm{~m}, 6 \mathrm{H}, 3 \times \operatorname{ring} \mathrm{CH}_{2}\right), 1.06$ 
(s, $\left.12 \mathrm{H}, 2 \times \mathrm{CMe}_{2}\right) ;{ }^{13} \mathrm{C}$ NMR $\left(100 \mathrm{MHz}, \mathrm{C}_{6} \mathrm{D}_{6}\right) 142.5(\mathrm{ArC}), 132.6(\mathrm{NCH}), 129.0(\mathrm{ArC}-\mathrm{H}), 128.7(\mathrm{ArC}-$ $\mathrm{H}), 126.2(\mathrm{NCH}=\mathrm{CH}), 123.4(\mathrm{ArC}-\mathrm{H}), 53.9\left(2 \times \mathrm{CMe}_{2}\right), 41.7\left(2 \times \mathrm{CMe}_{2} \mathrm{CH}_{2}\right), 37.5$ and $33.3\left(\mathrm{PhCH}_{2}\right.$ and $\left.=\mathrm{CHCH}_{2}\right), 28.0\left(2 \times \mathrm{CMe}_{2}\right), 18.2\left(\mathrm{CH}_{2}\right)$; HRMS $m / z\left(\mathrm{M}+\mathrm{H}^{+}\right)$Found: $272.2383 . \mathrm{C}_{19} \mathrm{H}_{30} \mathrm{~N}$ requires 272.2378

1-[5-(tert-Butyldimethylsilanyloxy)-pent-1-enyl]-2,2,6,6-tetramethylpiperidine $\mathbf{2 f}$

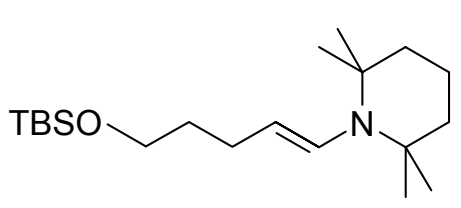

According to the general procedure, 1-tert-butyldimethylsilyoxy-4,5epoxypentane (513 $\mathrm{mg}, 2.37 \mathrm{mmol})$ gave enamine $\mathbf{2 f}$ as a colorless oil (620 mg, 77\%), IR ( $\left.\mathrm{cm}^{-1}\right) 2978 \mathrm{~s}, 2857 \mathrm{~s}, 1731 \mathrm{w}, 1661 \mathrm{~m}(\mathrm{C}=\mathrm{C}), 1641 \mathrm{~m}$ $(\mathrm{C}=\mathrm{C}), 1471 \mathrm{~s}, 1376 \mathrm{~s}, 1362 \mathrm{w}, 1255 \mathrm{~s}, 1175 \mathrm{~m}, 1131 \mathrm{~s}, 1099(\mathrm{C}-\mathrm{O}), 1035 \mathrm{~m}, 1006 \mathrm{~m} ;{ }^{1} \mathrm{H}$ NMR $(500 \mathrm{MHz}$, $\left.\mathrm{C}_{6} \mathrm{D}_{6}\right) 5.79(\mathrm{dt}, 1 \mathrm{H}, J=14$ and $1, \mathrm{NCH}), 5.24(\mathrm{dt}, 1 \mathrm{H}, J=14$ and $7,=\mathrm{CH}), 3.60\left(\mathrm{t}, 2 \mathrm{H}, J=7, \mathrm{OCH}_{2}\right)$, $2.12\left(\mathrm{dt}, 2 \mathrm{H}, J=7\right.$ and $\left.7,=\mathrm{CHCH}_{2}\right), 1.64-1.41\left(\mathrm{~m}, 8 \mathrm{H}, 4 \times \mathrm{CH}_{2}\right), 1.10\left(\mathrm{~s}, 12 \mathrm{H}, 2 \times \mathrm{CMe}_{2}\right), 0.96(\mathrm{~s}, 9 \mathrm{H}$, $\left.\mathrm{CMe}_{3}\right), 0.01\left(\mathrm{~s}, 6 \mathrm{H}, \mathrm{SiMe}_{2}\right) ;{ }^{13} \mathrm{C} \mathrm{NMR}\left(125 \mathrm{MHz}, \mathrm{C}_{6} \mathrm{D}_{6}\right) 132.1(\mathrm{NCH}), 124.8(=\mathrm{CH}), 62.8\left(\mathrm{OCH}_{2}\right), 53.7$ $\left(2 \times \mathrm{CMe}_{2}\right), 41.6\left(2 \times \mathrm{CMe}_{2} \mathrm{CH}_{2}\right), 34.0\left(=\mathrm{CHCH}_{2}\right), 28.0\left(2 \times \mathrm{CMe}_{2}\right), 27.3\left(\right.$ chain $\left.\mathrm{CH}_{2}\right), 26.2\left(\mathrm{CMe}_{3}\right)$, 18.6 (ring $\left.\mathrm{CH}_{2}\right), 18.1\left(\mathrm{SiCMe}_{3}\right),-5.0$ (SiMe), -5.1 (SiMe); HRMS m/z (M+ $\left.\mathrm{H}^{+}\right)$Found: 340.3035. $\mathrm{C}_{20} \mathrm{H}_{42} \mathrm{NOSi}$ requires 340.3036 .

1-(5-N-Boc- $N$-methylamino-pent-1-enyl)-2,2,6,6-tetramethylpiperidine $\mathbf{2 g}$

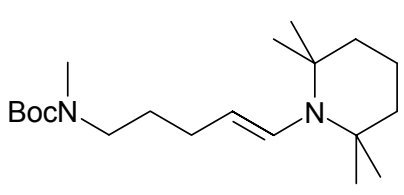
According to the general procedure, 5-( $N$-Boc- $N$-methylamino $)-1,2-$ epoxypentane $(210 \mathrm{mg}, 0.98 \mathrm{mmol})$ gave enamine $\mathbf{2 g}$ as a light yellow oil (209 mg, 63\%); IR $\left(\mathrm{cm}^{-1}\right) 3380 \mathrm{w}, 2927 \mathrm{~s}, 1693 \mathrm{~s}(\mathrm{C}=\mathrm{O}), 1640 \mathrm{~m}(\mathrm{C}=\mathrm{C}), 1452 \mathrm{~s}$, $1391 \mathrm{~s}, 1375 \mathrm{~s}, 1364 \mathrm{~s}, 1305 \mathrm{~s}, 1265 \mathrm{~s}, 1246 \mathrm{~s}, 1221 \mathrm{~s}, 1173 \mathrm{~s}, 1094 \mathrm{~m}, 1080 \mathrm{w}, 1061 \mathrm{~m}, 1035 \mathrm{~m}, 955 \mathrm{~s} ;{ }^{1} \mathrm{H}$ $\operatorname{NMR}\left(250 \mathrm{MHz}, \mathrm{C}_{6} \mathrm{D}_{6}, 60^{\circ} \mathrm{C}\right) 5.77(\mathrm{dt}, 1 \mathrm{H}, J=14$ and $1, \mathrm{NCH}), 5.17(\mathrm{dt}, 1 \mathrm{H}, J=14$ and $7,=\mathrm{CH})$, 3.15 (bt, 2H, $\left.J=7, \mathrm{NCH}_{2}\right), 2.67$ (bs, 3H, NMe), $1.95\left(\mathrm{dt}, 2 \mathrm{H}, J=7\right.$ and 7, $\left.=\mathrm{CHCH}_{2}\right), 1.67-1.37$ (m, $17 \mathrm{H}, 4 \times \mathrm{CH}_{2}$ and $\left.\mathrm{CMe}_{3}\right), 1.10\left(\mathrm{~s}, 12 \mathrm{H}, 2 \times \mathrm{CMe}_{2}\right) ;{ }^{13} \mathrm{C} \mathrm{NMR}\left(62.5 \mathrm{MHz}, \mathrm{C}_{6} \mathrm{D}_{6}, 60{ }^{\circ} \mathrm{C}\right) 155.6(\mathrm{C}=\mathrm{O})$, 
$132.4(\mathrm{NCH}), 123.4(=\mathrm{CH}), 78.7\left(\mathrm{OCMe}_{3}\right), 54.0\left(2 \times C \mathrm{Me}_{2}\right), 41.8,34.2,29.3,28.7,28.7,28.5,28.1$, 18.1 (ring $\left.\mathrm{CH}_{2}\right)$; HRMS $m / z\left(\mathrm{M}+\mathrm{H}^{+}\right)$Found: 339.3012. $\mathrm{C}_{20} \mathrm{H}_{39} \mathrm{~N}_{2} \mathrm{O}_{2}$ requires 339.3011 .

2,2,6,6-Tetramethyl-1-[4-(2,5,5-trimethyl-[1,3]dioxan-2-yl)-but-1-enyl]-piperidine $\mathbf{2 h}$

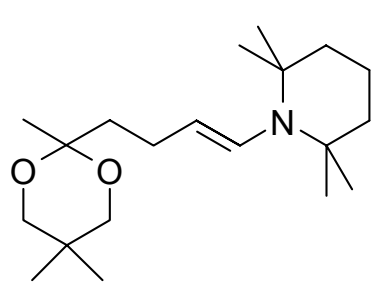

According to the general procedure, 2,5,5-trimethyl-2-(2-oxiranyl-ethyl)[1.3]dioxane (238 $\mathrm{mg}, 1.19 \mathrm{mmol})$ gave enamine $\mathbf{2 h}$ as a colorless oil (292 $\mathrm{mg}$, $76 \%)$; IR $\left(\mathrm{cm}^{-1}\right) 2928 \mathrm{~s}, 2867 \mathrm{~s}, 2361 \mathrm{w}, 2341 \mathrm{w}, 1729 \mathrm{w}, 1661 \mathrm{w}(\mathrm{C}=\mathrm{C}), 1641 \mathrm{~m}$ $(\mathrm{C}=\mathrm{C}), 1453 \mathrm{~m}, 1394 \mathrm{~m}, 1375 \mathrm{~s}, 1362 \mathrm{~s}, 1265 \mathrm{~s}, 1247 \mathrm{~s}, 1212 \mathrm{~s}, 1190 \mathrm{~m}, 1175 \mathrm{~m}$, $1130 \mathrm{~s}, 1091 \mathrm{~s}, 1042 \mathrm{~m}, 1022 \mathrm{~m} ;{ }^{1} \mathrm{H}$ NMR $\left(250 \mathrm{MHz}, \mathrm{C}_{6} \mathrm{D}_{6}\right) 5.81(\mathrm{dt}, 1 \mathrm{H}, J=14$ and $1, \mathrm{NCH}), 5.34(\mathrm{dt}$, $1 \mathrm{H}, J=14$ and $7, \mathrm{NCH}=\mathrm{CH}), 3.40-3.25\left(\mathrm{~m}, 4 \mathrm{H}, 2 \times \mathrm{OCH}_{2}\right), 2.47-2.29\left(\mathrm{~m}, 2 \mathrm{H}, \mathrm{O}_{2} \mathrm{CCH}_{2}\right), 1.96-1.82(\mathrm{~m}$, $\left.2 \mathrm{H},=\mathrm{CHCH}_{2}\right), 1.51-1.33\left(\mathrm{~m}, 6 \mathrm{H}, 3 \times \mathrm{CH}_{2}\right), 1.30(\mathrm{~s}, 3 \mathrm{H}, M e), 1.08\left(\mathrm{~s}, 12 \mathrm{H}, 2 \times \mathrm{CMe}_{2}\right), 0.91(\mathrm{~s}, 3 \mathrm{H}, M e)$, $0.60(\mathrm{~s}, 3 \mathrm{H}, \mathrm{Me}) ;{ }^{13} \mathrm{C} \mathrm{NMR}\left(62.5 \mathrm{MHz}, \mathrm{C}_{6} \mathrm{D}_{6}\right) 131.7(\mathrm{NCH}), 126.2(\mathrm{NCH}=\mathrm{CH}), 98.9\left(\mathrm{O}_{2} \mathrm{C}\right), 70.5(2 \times$ $\left.\mathrm{OCH}_{2}\right), 53.8\left(2 \times \mathrm{CMe}_{2}\right), 41.7\left(2 \times \mathrm{NCMe}_{2} \mathrm{CH}_{2}\right), 40.6,30.0,28.1\left(2 \times \mathrm{NCMe}_{2}\right), 25.4,23.1,22.6,20.2$, 18.2; HRMS $m / z\left(\mathrm{M}+\mathrm{H}^{+}\right)$Found: 324.2893. $\mathrm{C}_{20} \mathrm{H}_{38} \mathrm{NO}_{2}$ requires 324.2903.

1,8-Bis-(2,2,6,6-tetramethyl-piperidin-1-yl)-1,7-octadiene $\mathbf{2 i}$

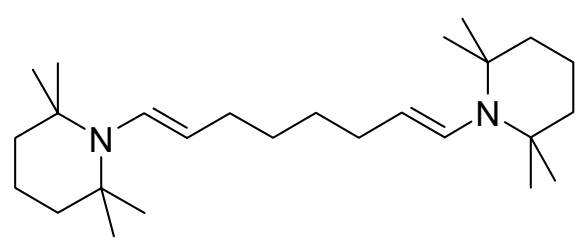

According to the general procedure, except employing 5.0 equiv. of LTMP, 1,2,7,8-diepoxyoctane (337 $\mathrm{mg}, 2.37 \mathrm{mmol})$ gave enamine $\mathbf{2 j}$ as a colorless oil (634 $\mathrm{mg}, 69 \%)$; IR $\left(\mathrm{cm}^{-1}\right) 2965 \mathrm{~s}$, 2926s, $1661 \mathrm{~m}(\mathrm{C}=\mathrm{C}), 1641 \mathrm{~m}(\mathrm{C}=\mathrm{C}), 1461 \mathrm{~m}, 1376 \mathrm{~s}, 1362 \mathrm{~s}, 1353 \mathrm{~m}, 1265 \mathrm{~s}, 1246 \mathrm{~s}, 1201 \mathrm{w}, 1175 \mathrm{~s}$, $1131 \mathrm{~s}, 1033 \mathrm{~m}, 955 \mathrm{~s} ;{ }^{1} \mathrm{H} \mathrm{NMR}\left(400 \mathrm{MHz}, \mathrm{C}_{6} \mathrm{D}_{6}\right) 5.77(\mathrm{dt}, 2 \mathrm{H}, J=14$ and $1,2 \times \mathrm{NCH}), 5.31(\mathrm{dt}, 2 \mathrm{H}, J=$ 14 and $7,2 \times \mathrm{NCH}=\mathrm{CH}), 2.17-1.99\left(\mathrm{~m}, 4 \mathrm{H}, 2 \times=\mathrm{CHCH}_{2}\right), 1.51-1.38\left(\mathrm{~m}, 16 \mathrm{H}, 6 \times\right.$ ring $\mathrm{CH}_{2}$ and $2 \times$ chain $\left.\mathrm{CH}_{2}\right), 1.12\left(24 \mathrm{H}, \mathrm{s}, 4 \times \mathrm{CMe}_{2}\right) ;{ }^{13} \mathrm{C} \mathrm{NMR}\left(100 \mathrm{MHz}, \mathrm{C}_{6} \mathrm{D}_{6}\right) 131.0(2 \times \mathrm{NCH}=), 126.6(2 \times$ $\mathrm{NCH}=\mathrm{CH}), 53.8\left(4 \times \mathrm{CMe}_{2}\right), 41.6\left(4 \times \mathrm{CMe}_{2} \mathrm{CH}_{2}\right), 30.9$ and $30.4\left(2 \times\right.$ chain $\mathrm{CH}_{2}$ and $\left.2 \times=\mathrm{CHC}\right), 29.0$ $\left(4 \times \mathrm{CMe}_{2}\right), 18.3\left(2 \times \operatorname{ring} \mathrm{CH}_{2}\right) ; \mathrm{HRMS} m / z\left(\mathrm{M}+\mathrm{H}^{+}\right)$Found: $389.3884 . \mathrm{C}_{26} \mathrm{H}_{49} \mathrm{~N}_{2}$ requires 389.3896 . 
tert-Butyl-hex-1-enyl-isopropylamine 3a

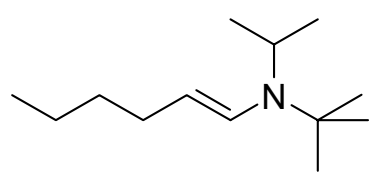

To a solution of $N$-tert-butylisopropylamine $\left(1.50 \mathrm{~cm}^{3}, 9.46 \mathrm{mmol}\right)$ in THF $\left(15 \mathrm{~cm}^{3}\right)$ at $-78{ }^{\circ} \mathrm{C}$ was added $n$-BuLi $\left(1.6 \mathrm{~mol} \mathrm{dm}{ }^{-3}\right.$ in hexanes; $5.91 \mathrm{~cm}^{3}, 9.46$ mmol) dropwise. The solution was allowed to warm to $25^{\circ} \mathrm{C}$ over $15 \mathrm{~min}$. before a solution of 1,2-epoxyhexane $(378 \mathrm{mg}, 3.78 \mathrm{mmol})$ in THF $\left(5 \mathrm{~cm}^{3}\right)$ was added in one portion. The reaction was stirred at room temperature for $1 \mathrm{~h}$ before being filtered through a pad of celite $\left(5 \mathrm{~cm}^{2}\right.$ $\times 5 \mathrm{~cm})$. The pad was washed with $\mathrm{Et}_{2} \mathrm{O}\left(250 \mathrm{~cm}^{3}\right)$. The solvent was removed in vacuo, and the residue purified by bulb-to-bulb distillation to give enamine 3a (314 mg, 42\%) as a colourless oil; bp $90{ }^{\circ} \mathrm{C} /$ $0.1 \mathrm{mbar} ;$ IR $\left(\mathrm{cm}^{-1}\right) 2970 \mathrm{~s}, 2925 \mathrm{~s}, 2873 \mathrm{~s}, 1645 \mathrm{~s}(\mathrm{C}=\mathrm{C}), 1465 \mathrm{w}, 1377 \mathrm{~m}, 1363 \mathrm{~m}, 1302 \mathrm{~m}, 1220 \mathrm{~m} ;{ }^{1} \mathrm{H}$ $\operatorname{NMR}(400 \mathrm{MHz}) 5.93(\mathrm{dt}, 1 \mathrm{H}, J=14$ and $1, \mathrm{NCH}=), 4.48(\mathrm{dt}, 1 \mathrm{H}, J=14$ and 7, =CH), $3.56(\mathrm{dsept}, 1 \mathrm{H}$, $J=14$ and 1, $\mathrm{CHMe}$ ), 1.99-1.93 (m, 2H, $\left.=\mathrm{CHCH}_{2}\right), 1.35-1.30\left(\mathrm{~m}, 4 \mathrm{H}, 2 \times \mathrm{CH}_{2}\right), 1.23-1.18(\mathrm{~m}, 15 \mathrm{H}$, $\mathrm{CMe}_{3}$ and $\left.\mathrm{CMe} 2\right), 0.91(\mathrm{t}, 3 \mathrm{H}, J=7, \mathrm{Me}) ;{ }^{13} \mathrm{C} \mathrm{NMR}(100 \mathrm{MHz}) 129.9(\mathrm{NCH}=), 105.3(=C \mathrm{H}), 55.5$ (NC), $46.1(\mathrm{NC}), 33.8\left(\mathrm{CH}_{2}\right), 31.5\left(\mathrm{CH}_{2}\right), 29.0\left(\mathrm{CMe}_{3}\right), 22.1\left(\mathrm{CH}_{2}\right), 20.9\left(\mathrm{CMe}_{2}\right), 14.0(\mathrm{Me}) ; \mathrm{HRMS} m / z$ $\left(\mathrm{M}+\mathrm{H}^{+}\right)$Found: 198.2228. $\mathrm{C}_{13} \mathrm{H}_{28} \mathrm{~N}$ requires 198.2222.

tert-Butyl-(hex-1-enyl)-(1,2,2-trimethyl-propyl)-amine 3b

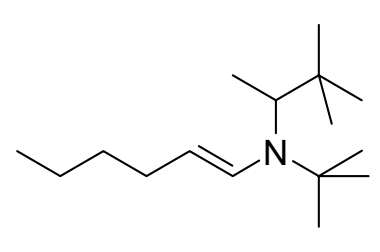

To a solution of $N$-tert-butylpinacoylamine $(1.00 \mathrm{~g}, 6.37 \mathrm{mmol})$ in THF (15 $\left.\mathrm{cm}^{3}\right)$ at $-78{ }^{\circ} \mathrm{C}$ was added $n$-BuLi $\left(1.6 \mathrm{~mol} \mathrm{dm}{ }^{-3}\right.$ in hexanes; $3.98 \mathrm{~cm}^{3}, 6.37$ mmol) dropwise. The solution was allowed to warm to $25^{\circ} \mathrm{C}$ over $15 \mathrm{~min}$. before a solution of 1,2-epoxyhexane $(255 \mathrm{mg}, 2.55 \mathrm{mmol})$ in THF $\left(5 \mathrm{~cm}^{3}\right)$ was added in one portion. The reaction was stirred at room temperature for $1 \mathrm{~h}$ before being filtered through a pad $\left(5 \mathrm{~cm}^{2} \times 5 \mathrm{~cm}\right)$ of celite. The pad was washed with $\mathrm{Et}_{2} \mathrm{O}\left(250 \mathrm{~cm}^{3}\right)$. The solvent was removed in vacuo, and the residue purified by bulb-to-bulb distillation to give enamine 3a (353 mg, 58\%) as a colourless oil; bp $125{ }^{\circ} \mathrm{C} / 0.03$ mbar; IR $\left(\mathrm{cm}^{-1}\right)$ 2957s, 2924s, $1646(\mathrm{C}=\mathrm{C}), 1457 \mathrm{~m}, 1370 \mathrm{~m}, 1259 \mathrm{w}, 1195 \mathrm{w} ;{ }^{1} \mathrm{H}$ NMR $(500$ $\left.\mathrm{MHz}, \mathrm{C}_{6} \mathrm{D}_{6}\right) 5.83(\mathrm{dt}, 1 \mathrm{H}, J=14$ and $1, \mathrm{NCH}=), 4.94(\mathrm{dt}, 1 \mathrm{H}, J=14$ and 7, =CH), $2.92(\mathrm{q}, 1 \mathrm{H}, J=7$, $\mathrm{CHMe}), 2.05\left(\mathrm{dt}, 2 \mathrm{H}, J=7\right.$ and 7, $\left.=\mathrm{CHCH}_{2}\right), 1.42-1.35\left(\mathrm{~m}, 4 \mathrm{H}, 2 \times \mathrm{CH}_{2}\right), 1.15\left(\mathrm{~s}, 9 \mathrm{H}, \mathrm{NCMe}_{3}\right), 1.05(\mathrm{~d}$, 
$3 \mathrm{H}, J=7, \mathrm{CHMe}), 0.96(\mathrm{~s}, 9 \mathrm{H}, \mathrm{CHCMe}), 0.91(\mathrm{t}, 3 \mathrm{H}, J=7, \mathrm{Me}) ;{ }^{13} \mathrm{C} \mathrm{NMR} 133.0(\mathrm{NCH}=), 117.3$ $(=\mathrm{CH}), 58.6(\mathrm{NCH}), 55.5(\mathrm{NC}), 36.3\left(\mathrm{CHCMe}_{3}\right), 33.5\left(=\mathrm{CHCH}_{2}\right), 31.5\left(\mathrm{CH}_{2}\right), 29.3\left(\mathrm{CMe}_{3}\right), 28.6$ $\left(\mathrm{CMe}_{3}\right), 22.7\left(\mathrm{CH}_{2}\right), 14.2(\mathrm{Me}), 14.2(\mathrm{Me})$; HRMS $m / z\left(\mathrm{M}+\mathrm{H}^{+}\right)$Found: $240.2692 . \mathrm{C}_{16} \mathrm{H}_{34} \mathrm{~N}$ requires 240.2691.

\section{General procedure for enamine alkylation:}

2-Benzylhexanal ${ }^{2} \mathbf{4 a}$

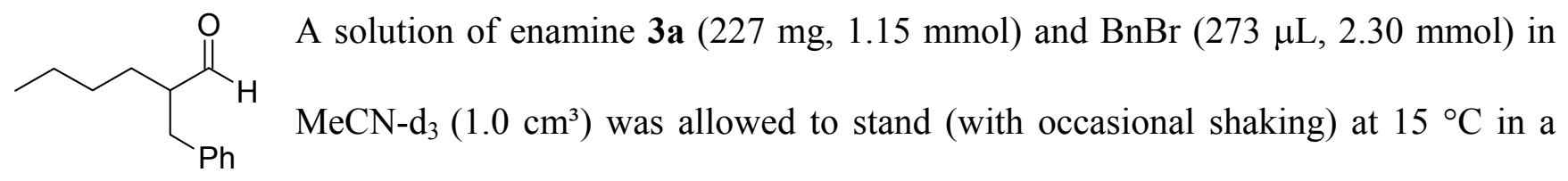

NMR tube fitted with a PTFE valve, ${ }^{3}$ until consumption of enamine 3a was judged complete by ${ }^{1} \mathrm{H}$ NMR spectroscopy (18 h). Acidic buffer solution (made up of AcOH (0.5 g), AcONa (0.5g) and water $(1.0 \mathrm{~g}))\left(0.5 \mathrm{~cm}^{3}\right)$ was added and the mixture was allowed to stand at the same temperature as before, for $1 \mathrm{~h}$ with occasional shaking before being separated between $\mathrm{H}_{2} \mathrm{O}\left(10 \mathrm{~cm}^{3}\right)$ and $\mathrm{Et}_{2} \mathrm{O}\left(10 \mathrm{~cm}^{3}\right)$. The aqueous phase was washed with $\mathrm{Et}_{2} \mathrm{O}\left(10 \mathrm{~cm}^{3}\right)$ and the combined organic layers were washed with brine $\left(20 \mathrm{~cm}^{3}\right)$, dried $\left(\mathrm{MgSO}_{4}\right)$, filtered and the solvent removed in vacuo. Purification by column chromatography $\left(\mathrm{SiO}_{2}, 5 \% \mathrm{Et}_{2} \mathrm{O} /\right.$ light petrol) gave 2-benzylhexanal $4 \mathbf{4}(217 \mathrm{mg}$, quant.) as a colorless oil; $\mathrm{R}_{f} 0.26\left(5 \% \mathrm{Et}_{2} \mathrm{O} /\right.$ light petrol); IR ( $\left.\mathrm{cm}^{-1}\right) 3086 \mathrm{w}, 3063 \mathrm{w}, 3028 \mathrm{~m}, 2957 \mathrm{~s}, 2931 \mathrm{~s}, 2859 \mathrm{~s}, 2711 \mathrm{~m}$, 2360w, 2340w, 1725s (C=O), 1604w, 1496m, 1466m, 1454s, 1392w, 1379w, 1030w; ${ }^{1} \mathrm{H}$ NMR (400 MHz) $9.67(\mathrm{~d}, 1 \mathrm{H}, J=3, \mathrm{CHO}), 7.33-7.26(\mathrm{~m}, 2 \mathrm{H}, 2 \times \mathrm{ArC}-\mathrm{H}), 7.25-7.15(\mathrm{~m}, 3 \mathrm{H}, 3 \times \mathrm{ArC}-\mathrm{H}), 2.99$ and $2.73\left(\right.$ AB-part of $\left.\mathrm{ABX}, 2 \mathrm{H}, J_{\mathrm{AX}}=7, J_{\mathrm{BX}}=7, J_{\mathrm{AB}}=14, \mathrm{CH}_{2} \mathrm{Ph}\right), 2.67-2.58(\mathrm{~m}, 1 \mathrm{H}, \mathrm{CHCHO}), 1.72-1.22$ (m, 6H, $\left.\left(\mathrm{CH}_{2}\right)_{3} \mathrm{Me}\right), 0.89$ (t, 3H, $\left.J=7, \mathrm{Me}\right) ;{ }^{13} \mathrm{C} \mathrm{NMR}(100 \mathrm{MHz}) 204.8$ (CHO), 138.9 (ArC), 129.0 (2 $\times$ ArC-H $), 128.5(2 \times$ ArC-H $), 126.3(\mathrm{ArC}-\mathrm{H}) 53.4(\mathrm{CHCHO}), 35.0\left(\mathrm{CH}_{2} \mathrm{Ph}\right), 29.1\left(\mathrm{CH}_{2}\right), 28.3\left(\mathrm{CH}_{2}\right)$ $22.7\left(\mathrm{CH}_{2}\right), 13.8(\mathrm{Me})$. 
2-Allylhexanal ${ }^{4} \mathbf{4 b}$

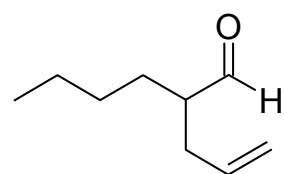

According to the general procedure, a solution of enamine $\mathbf{3 a}(219 \mathrm{mg}, 1.11 \mathrm{mmol})$

and allyl bromide (192 $\mu \mathrm{L}, 2.22 \mathrm{mmol})$ gave 2-allylhexanal 4b as a colorless oil (149mg, 96\%); $\mathrm{R}_{f} 0.23$ (5\% Et $2 \mathrm{O} /$ light petrol); IR ( $\left.\mathrm{cm}^{-1}\right) 3080 \mathrm{w}, 2959 \mathrm{~s}, 2931 \mathrm{~s}, 2861 \mathrm{~m}, 2708 \mathrm{w}, 1728 \mathrm{~s}$ $(\mathrm{C}=\mathrm{O}), 1642 \mathrm{w}, 1457 \mathrm{w}, 1440 \mathrm{w} ;{ }^{1} \mathrm{H}$ NMR $(400 \mathrm{MHz}) 9.61(\mathrm{~d}, 1 \mathrm{H}, J=3, \mathrm{CHO}), 5.79-5.68(\mathrm{~m}, 1 \mathrm{H}$, $=\mathrm{CH}), 5.10-5.03\left(\mathrm{~m}, 2 \mathrm{H},=\mathrm{CH}_{2}\right), 2.43-2.31\left(\mathrm{~m}, 2 \mathrm{H},=\mathrm{CHCH}_{2}\right), 2.28-2.18(\mathrm{~m}, 1 \mathrm{H}, \mathrm{CHCHO}), 1.69-1.24$ (m, 6H, $\left.\left(\mathrm{CH}_{2}\right)_{3} \mathrm{Me}\right), 0.91(\mathrm{t}, 3 \mathrm{H}, J=7, \mathrm{Me}) ;{ }^{13} \mathrm{C} \mathrm{NMR}$ (100 MHz) $204.9(\mathrm{CHO}), 135.0(=\mathrm{CH}), 117.0$ $\left(=\mathrm{CH}_{2}\right), 51.2(\mathrm{CHCHO}), 33.0(=\mathrm{CHCH} 2), 29.0\left(\mathrm{CH}_{2}\right), 28.0\left(\mathrm{CH}_{2}\right), 22.7\left(\mathrm{CH}_{2}\right), 13.9(\mathrm{Me})$.

Methyl-3-formylheptanoate ${ }^{5}$ 4c

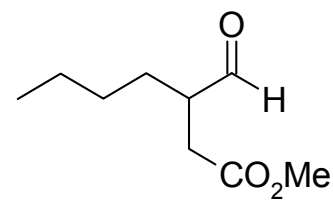

According to the general procedure, a solution of enamine 3a $(212 \mathrm{mg}, 1.07$ mmol) and $\alpha$-bromo methyl acetate $(198 \mu \mathrm{L}, 2.14 \mathrm{mmol})$ at $15{ }^{\circ} \mathrm{C}$ for $16 \mathrm{~h}$ gave methyl-3-formylheptanoate $4 \mathrm{c}(167 \mathrm{mg}, 91 \%)$ as a colorless oil; $\mathrm{R}_{f} 0.20(15 \%$ $\mathrm{Et}_{2} \mathrm{O} /$ light petrol); IR $\left(\mathrm{cm}^{-1}\right) 2957 \mathrm{~s}, 2233 \mathrm{~s}, 2862 \mathrm{~s}, 2722 \mathrm{w}, 1738 \mathrm{bs}(\mathrm{C}=\mathrm{O}), 1460 \mathrm{~m}, 1438 \mathrm{~s}, 1414 \mathrm{~m}$, 1394m, 1365m, 1206s, 1174s, 1111w, 1018m; ${ }^{1} \mathrm{H}$ NMR (400 MHz) 9.70 (d, 1H, J=1, CHO), 3.67 (s, $3 \mathrm{H}, \mathrm{OMe}), 2.86-2.77(\mathrm{~m}, 1 \mathrm{H}, \mathrm{CHCHO}), 2.69$ and $2.39\left(\mathrm{AB}-\right.$ part of $\mathrm{ABX}, 2 \mathrm{H}, J_{\mathrm{AX}}=5, J_{\mathrm{BX}}=8, J_{\mathrm{AB}}=$ 16, $\left.\mathrm{CH}_{2} \mathrm{CO}_{2}\right), 1.79-1.66(\mathrm{~m}, 1 \mathrm{H}, \mathrm{CH}(\mathrm{H}) \mathrm{CH}), 1.53-1.40(\mathrm{~m}, 1 \mathrm{H}, \mathrm{CH}(H) \mathrm{CH}), 1.39-1.25(\mathrm{~m}, 4 \mathrm{H}$, $\left.\left(\mathrm{CH}_{2}\right)_{2} \mathrm{Me}\right), 0.89$ (t, 3H, $\left.J=7, \mathrm{Me}\right) ;{ }^{13} \mathrm{C} \mathrm{NMR}(100 \mathrm{MHz}) 202.9(\mathrm{CHO}), 172.4\left(\mathrm{CO}_{2}\right), 51.8(\mathrm{CHCHO})$, 47.6 $\left(\mathrm{CH}_{2} \mathrm{CO}_{2} \mathrm{Me}\right), 32.7\left(\mathrm{CH}_{2}\right), 28.8\left(\mathrm{CH}_{2}\right), 28.2\left(\mathrm{CH}_{2}\right), 22.6\left(\mathrm{CH}_{2}\right), 13.8(\mathrm{Me})$.

\section{2-Prop-2-ynylhexanal ${ }^{6} \mathbf{4 d}$}

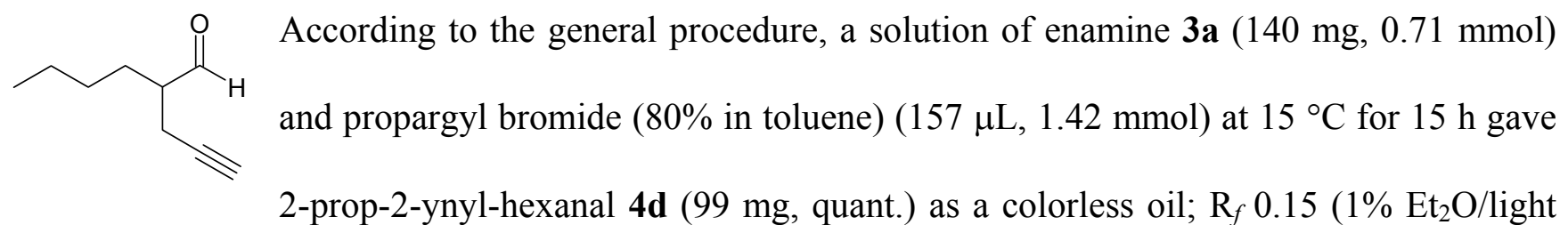

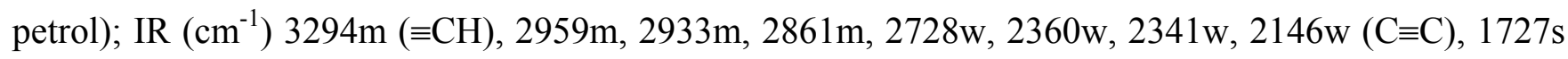
$(\mathrm{C}=\mathrm{O}), 1467 \mathrm{w}, 1380 \mathrm{w} ;{ }^{1} \mathrm{H}$ NMR (400 MHz) $9.68(\mathrm{~d}, 1 \mathrm{H}, J=1, \mathrm{CHO}), 2.52-2.36(\mathrm{~m}, 3 \mathrm{H}, \mathrm{CHCHO}$ and 
$\left.\mathrm{CH}_{2} \mathrm{C} \equiv \mathrm{CH}\right), 2.01-1.98(\mathrm{t}, 1 \mathrm{H}, J=3, \mathrm{C} \equiv \mathrm{CH}), 1.84-1.71(\mathrm{~m}, 1 \mathrm{H}, \mathrm{CH}(\mathrm{H}) \mathrm{CH}), 1.69-1.56(\mathrm{~m}, 1 \mathrm{H}$,

$\mathrm{CH}(\mathrm{H}) \mathrm{CH}), 1.40-1.26\left(\mathrm{~m}, 4 \mathrm{H},\left(\mathrm{CH}_{2}\right)_{2} \mathrm{Me}\right), 0.90(\mathrm{t}, 3 \mathrm{H}, J=7, \mathrm{Me}) ;{ }^{13} \mathrm{C} \mathrm{NMR}(100 \mathrm{MHz}) 203.2(\mathrm{CHO})$, $80.9(\mathrm{C} \equiv \mathrm{CH}), 70.2(\mathrm{C} \equiv \mathrm{CH}), 50.0(\mathrm{CHCHO}), 28.6\left(\mathrm{CH}_{2}\right), 27.6\left(\mathrm{CH}_{2} \mathrm{C} \equiv\right), 22.6\left(\mathrm{CH}_{2}\right), 17.7\left(\mathrm{CH}_{2}\right), 13.8$ $(\mathrm{Me})$.

\section{4-Formyl-octanenitrile 4e}

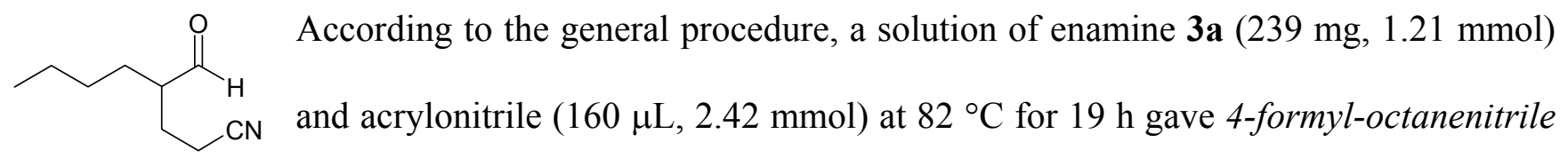
4e (169 mg, $91 \%)$ as a colorless oil; $\mathrm{R}_{f} 0.32\left(30 \% \mathrm{Et}_{2} \mathrm{O} /\right.$ light petrol); IR ( $\left.\mathrm{cm}^{-1}\right) 2958 \mathrm{~s}, 2933 \mathrm{~s}, 2861 \mathrm{~s}$,

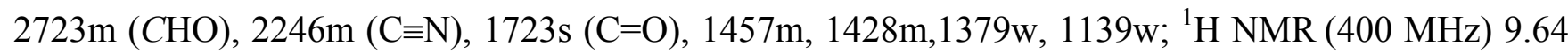
(d, $1 \mathrm{H}, J=1, \mathrm{CHO}), 2.52-2.32\left(\mathrm{~m}, 3 \mathrm{H}, \mathrm{CHCHO}\right.$ and $\left.\mathrm{CH}_{2} \mathrm{C} \equiv \mathrm{N}\right), 2.07-1.95\left(\mathrm{~m}, 1 \mathrm{H}, 1\right.$ of $\left.\left(\mathrm{CH}_{2}\right)_{2} \mathrm{CH}\right) 1.79$ $1.65\left(\mathrm{~m}, 2 \mathrm{H}, 2\right.$ of $\left.\left(\mathrm{CH}_{2}\right)_{2} \mathrm{CH}\right), 1.56-1.44\left(\mathrm{~m}, 1 \mathrm{H}, 1\right.$ of $\left.\left(\mathrm{CH}_{2}\right)_{2} \mathrm{CH}\right), 1.39-1.25\left(\mathrm{~m}, 4 \mathrm{H},\left(\mathrm{CH}_{2}\right)_{2} \mathrm{Me}\right), 0.90(\mathrm{t}$, $3 \mathrm{H}, J=7, \mathrm{Me}) ;{ }^{13} \mathrm{C}$ NMR (100 MHz) $203.1(\mathrm{CHO}), 119.1(\mathrm{CN}), 50.1(\mathrm{CHCHO}), 28.7\left(\mathrm{CH}_{2}\right), 28.2$ $\left(\mathrm{CH}_{2}\right), 23.8\left(\mathrm{CH}_{2}\right), 22.6\left(\mathrm{CH}_{2}\right), 15.1\left(\mathrm{CH}_{2} \mathrm{CN}\right), 13.7(\mathrm{Me})$; HRMS $m / z\left(\mathrm{M}+\mathrm{NH}^{+}\right)$Found: 171.1504. $\mathrm{C}_{9} \mathrm{H}_{19} \mathrm{~N}_{2} \mathrm{O}$ requires 171.1497 .

\section{Methyl-4-formyloctanoate $\mathbf{4 f}$}

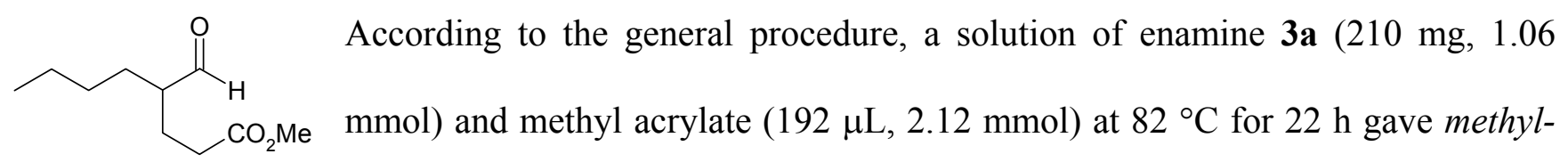
4-formyloctanoate $4 \mathbf{f}(139 \mathrm{mg}, 70 \%)$ as a colorless oil; $\mathrm{R}_{f} 0.24\left(15 \% \mathrm{Et}_{2} \mathrm{O} /\right.$ light petrol); IR (cm $\left.{ }^{-1}\right) 2986 \mathrm{~s}$, 2932s, 2861s, 2716m (CHO), 1738s (C=O), 1437s, 1375m, 1198s, 1171s, $1115 \mathrm{w}, 1011 \mathrm{w} ;{ }^{1} \mathrm{H}$ NMR (400 MHz) $9.56(\mathrm{~d}, 1 \mathrm{H}, J=3, \mathrm{CHO}), 3.63(\mathrm{~s}, 3 \mathrm{H}, \mathrm{OMe}), 2.38-2.22\left(3 \mathrm{H}, \mathrm{m}, \mathrm{CHCHO}\right.$ and $\left.\mathrm{CH}_{2} \mathrm{CO}_{2}\right), 1.99-$ $1.18\left(\mathrm{~m}, 8 \mathrm{H}, 4 \times \mathrm{CH}_{2}\right), 0.86(\mathrm{t}, 3 \mathrm{H}, J=7, \mathrm{Me}) ;{ }^{13} \mathrm{C} \mathrm{NMR}(100 \mathrm{MHz}) 204.3(\mathrm{CHO}), 173.4\left(\mathrm{CO}_{2}\right), 51.6$ (OMe), $51.0(\mathrm{CHCHO}), 31.3\left(\mathrm{CH}_{2}\right), 28.9\left(\mathrm{CH}_{2}\right), 28.4\left(\mathrm{CH}_{2}\right), 23.5\left(\mathrm{CH}_{2}\right), 22.6\left(\mathrm{CH}_{2}\right), 13.8(\mathrm{Me})$; HRMS $m / z\left(\mathrm{M}+\mathrm{NH}_{4}{ }^{+}\right)$Found: 204.1599. $\mathrm{C}_{10} \mathrm{H}_{22} \mathrm{NO}_{3}$ requires 204.1600. 
2-Methylhexanal ${ }^{7} \mathbf{4 g}$

According to the general procedure, a solution of enamine 3a $(227 \mathrm{mg}, 1.15 \mathrm{mmol})$ colourless oil (113mg, 86\%); $\mathrm{R}_{f} 0.34$ (5\% Et $2 \mathrm{O} /$ light petrol); IR ( $\mathrm{cm}^{-1}$ ) 2960s, 2932s, 2860m, 2706w, 2360w, 1728s (C=O), 1460m, 1379w; ${ }^{1} \mathrm{H}$ NMR (400 MHz) $9.61(\mathrm{~d}, 1 \mathrm{H}, J=2, \mathrm{CHO}), 2.37-2.28(\mathrm{~m}, 1 \mathrm{H}$, $\mathrm{CHMe}), 1.75-1.66(\mathrm{~m}, 1 \mathrm{H}, \mathrm{CH}(\mathrm{H}) n-\mathrm{Pr}), 1.43-1.23\left(\mathrm{~m}, 5 \mathrm{H},\left(\mathrm{CH}_{2}\right)_{2} \mathrm{Me}\right.$ and $\left.\mathrm{CH}(H) n-\mathrm{Pr}\right), 1.08(\mathrm{~d}, 3 \mathrm{H}, J=$ 7, $\mathrm{CHMe}), 0.90$ (t, 3H, $J=7, \mathrm{Me}) ;{ }^{13} \mathrm{C} \mathrm{NMR}(100 \mathrm{MHz}) 205.4(\mathrm{CHO}), 46.3(\mathrm{CHMe}), 30.2\left(\mathrm{CH}_{2}\right), 29.1$ $\left(\mathrm{CH}_{2}\right), 22.7\left(\mathrm{CH}_{2}\right), 13.9(\mathrm{Me}), 13.3(\mathrm{Me})$.

\section{2-Ethylhexanal ${ }^{3} \mathbf{4 h}$}

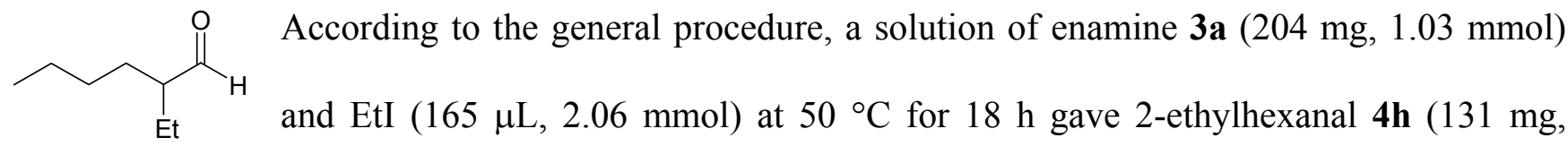
quant.) as a colorless oil; $\mathrm{R}_{f} 0.24$ (5\% Et ${ }_{2} \mathrm{O} /$ light petrol); IR $\left(\mathrm{cm}^{-1}\right) 2961 \mathrm{~s}, 2932 \mathrm{~s}, 2874 \mathrm{~m}, 2861 \mathrm{~m}$, 2703w, 1727s (C=O), 1461m, 1381m; ${ }^{1} \mathrm{H}$ NMR (400 MHz) 9.55 (d, 1H, J=3, CHO), 2.21-2.11 (m, 1H, CHEt), $1.70-1.38\left(\mathrm{~m}, 4 \mathrm{H}, 2 \times \mathrm{CH}_{2}\right), 1.37-1.19\left(\mathrm{~m}, 4 \mathrm{H}, 2 \times \mathrm{CH}_{2}\right), 0.93-0.86(\mathrm{~m}, 6 \mathrm{H}, 2 \times \mathrm{Me}) ;{ }^{13} \mathrm{C} \mathrm{NMR}$ (100 MHz) 205.7 (CHO), 53.4 (CHEt), $29.1\left(\mathrm{CH}_{2}\right), 28.1\left(\mathrm{CH}_{2}\right) 22.7\left(\mathrm{CH}_{2}\right), 21.8\left(\mathrm{CH}_{2}\right), 13.8(\mathrm{Me}), 11.4$ (Me).

2-n-Butylhexanal ${ }^{3} \mathbf{4 i}$

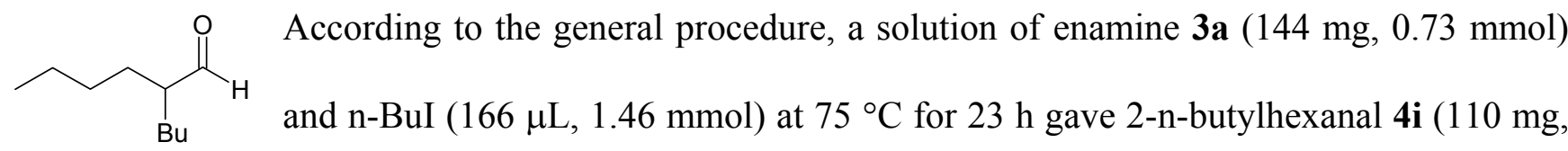
97\%.) as a colorless oil; $\mathrm{R}_{f} 0.21\left(1 \% \mathrm{Et}_{2} \mathrm{O} /\right.$ light petrol); IR ( $\left.\mathrm{cm}^{-1}\right) 2958 \mathrm{~s}, 2931 \mathrm{~s}, 2873 \mathrm{~s}, 2860 \mathrm{~s}, 2693 \mathrm{w}$, 1727s $(\mathrm{C}=\mathrm{O}), 1467 \mathrm{~m}, 1379 \mathrm{w} ;{ }^{1} \mathrm{H}$ NMR (400 MHz) $9.54(\mathrm{~d}, 1 \mathrm{H}, J=3, \mathrm{CHO}), 2.26-2.16(\mathrm{~m}, 1 \mathrm{H}$, $\left.\mathrm{CHBu}_{2}\right), 1.66-1.55(\mathrm{~m}, 2 \mathrm{H}, 2 \times \mathrm{CH}(\mathrm{H}) n-\mathrm{Pr}), 1.48-1.37(\mathrm{~m}, 2 \mathrm{H}, 2 \times \mathrm{CH}(H) n-\mathrm{Pr}), 1.36-1.19(\mathrm{~m}, 8 \mathrm{H}, 2 \times$ $\left.\left(\mathrm{CH}_{2}\right)_{2} \mathrm{Me}\right), 0.88(\mathrm{t}, 6 \mathrm{H}, J=7,2 \times \mathrm{Me}) ;{ }^{13} \mathrm{C} \mathrm{NMR}(100 \mathrm{MHz}) 205.6(\mathrm{CHO}), 51.9\left(\mathrm{CHBu}_{2}\right), 29.2(2 \times$ $\left.\mathrm{CH}_{2}\right), 28.6\left(2 \times \mathrm{CH}_{2}\right) 22.7\left(2 \times \mathrm{CH}_{2}\right), 13.8(2 \times \mathrm{Me})$. 
Similarly, a solution of enamine $\mathbf{3 b}(119 \mathrm{mg}, 0.50 \mathrm{mmol})$ and $n$-BuI $(113 \mu \mathrm{L}, 1.00 \mathrm{mmol})$ at $82{ }^{\circ} \mathrm{C}$ for 96 h gave 2-n-butylhexanal $4 \mathbf{i}(65 \mathrm{mg}, 84 \%)$ as a colorless oil; data as above.

\section{2-Butyldodecanal 4j}

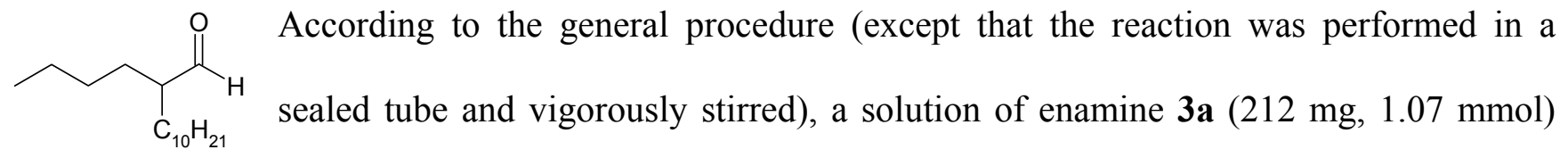
and 1-iododecane $(458 \mu \mathrm{L}, 2.14 \mathrm{mmol})$ at $82{ }^{\circ} \mathrm{C}$ for $22 \mathrm{~h}$ gave 2 -n-butyldodecanal $\mathbf{4 j}$ as a colorless oil (244 mg, 95\%); $\mathrm{R}_{f} 0.29$ (2\% Et $2 \mathrm{O} /$ light petrol); IR ( $\left.\mathrm{cm}^{-1}\right) 3434 \mathrm{w}, 2926 \mathrm{~s}, 2855 \mathrm{~s}, 2692 \mathrm{~m}(\mathrm{CHO}), 1728 \mathrm{~s}$ $(\mathrm{C}=\mathrm{O}), 1466 \mathrm{~s}, 1378 \mathrm{~m}, 1239 \mathrm{~m}, 1143 \mathrm{w}, 1016 \mathrm{w} ;{ }^{1} \mathrm{H}$ NMR (400 MHz) $9.54(\mathrm{~d}, 1 \mathrm{H}, J=3, \mathrm{CHO}), 2.26-$ $2.16(\mathrm{~m}, 1 \mathrm{H}, \mathrm{CHCHO}), 1.67-1.16\left(\mathrm{~m}, 24 \mathrm{H}, 12 \times \mathrm{CH}_{2}\right), 0.92-0.83(\mathrm{~m}, 6 \mathrm{H}, 2 \times \mathrm{Me}) ;{ }^{13} \mathrm{C} \mathrm{NMR}(100$ MHz) 205.7 (CHO), $51.9(\mathrm{CHCHO}), 31.9\left(\mathrm{CH}_{2}\right), 29.7\left(\mathrm{CH}_{2}\right), 29.6\left(\mathrm{CH}_{2}\right), 29.5\left(\mathrm{CH}_{2}\right), 29.4\left(\mathrm{CH}_{2}\right), 29.3$ $\left(\mathrm{CH}_{2}\right), 29.2\left(\mathrm{CH}_{2}\right), 28.9\left(\mathrm{CH}_{2}\right), 28.6\left(\mathrm{CH}_{2}\right), 27.0\left(\mathrm{CH}_{2}\right), 22.7\left(\mathrm{CH}_{2}\right), 22.6\left(\mathrm{CH}_{2}\right), 14.0(\mathrm{Me}), 13.8(\mathrm{Me})$; HRMS $m / z\left(\mathrm{M}+\mathrm{NH}_{4}{ }^{+}\right)$Found: 258.2801. $\mathrm{C}_{16} \mathrm{H}_{36} \mathrm{NO}$ requires 258.2797.

\section{2-Isopropylhexanal ${ }^{8} \mathbf{4 k}$}

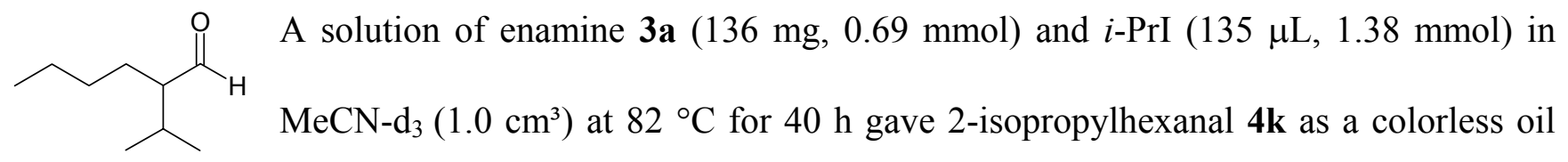
(79 mg, 80\%); $\mathrm{R}_{f} 0.29$ (2\% Et $2 \mathrm{O} /$ light petrol); IR ( $\left.\mathrm{cm}^{-1}\right) 2961 \mathrm{~s}, 2873 \mathrm{~s}, 1725 \mathrm{~s}(\mathrm{C}=\mathrm{O}), 1466 \mathrm{w}, 1371 \mathrm{w}$, 1264w, 1128w, 1066w; ${ }^{1} \mathrm{H}$ NMR (400 MHz) 9.61 (d, 1H, $\left.J=3, \mathrm{CHO}\right), 2.06-1.91$ (m, 2H, CHCHO and $\left.\mathrm{CHMe}_{2}\right), 1.69-1.57(\mathrm{~m}, 1 \mathrm{H}, \mathrm{CH}(\mathrm{H}) n-\mathrm{Pr}), 1.51-1.40(\mathrm{~m}, 1 \mathrm{H}, \mathrm{CH}(H) n-\mathrm{Pr}), 1.37-1.13\left(\mathrm{~m}, 4 \mathrm{H},\left(\mathrm{CH}_{2}\right)_{2} \mathrm{Me}\right)$, $0.96(\mathrm{~d}, 6 \mathrm{H}, J=7, \mathrm{CHMe} 2), 0.89(\mathrm{t}, 3 \mathrm{H}, J=7, \mathrm{Me}) ;{ }^{13} \mathrm{C}$ NMR $(100 \mathrm{MHz}) 206.1(\mathrm{CHO}), 58.3$ (CHCHO), $29.8\left(\mathrm{CH}_{2}\right), 28.3\left(\mathrm{CMe}_{2}\right), 25.8\left(\mathrm{CH}_{2}\right), 22.8\left(\mathrm{CH}_{2}\right), 20.2(\mathrm{CMe}(\mathrm{Me})), 19.8(\mathrm{CMe}(\mathrm{Me})), 13.9$ (Me); HRMS $m / z\left(\mathrm{M}+\mathrm{NH}_{4}^{+}\right)$Found: 160.1700. $\mathrm{C}_{9} \mathrm{H}_{2} \mathrm{NO}$ requires 160.1701. 
Similarly, a solution of enamine $3 \mathbf{b}(198 \mathrm{mg}, 0.83 \mathrm{mmol})$ and $i$-PrI $(248 \mu \mathrm{L}, 2.49 \mathrm{mmol})$ at $82{ }^{\circ} \mathrm{C}$ for 96

h gave 2-isopropylhexanal $\mathbf{4 k}$ as a colorless oil (58 mg, 49\%); data as above.

\section{References}

1 The synthesis of $\mathrm{N}$-tert-butylpinacoylamine from the same starting materials via a Leuckart-Wallach reaction has been reported to proceed in 23\% yield: Salvadori, P.; Rosini, C.; Lazzaroni, R.; Pini, D. J. Chem. Soc. Perkin Trans. 2, 1983, 1919-1922.

2 Bremand, N.; Normant, J. F.; Mangeney, P. Synlett.; 2000, 4, 532-534.

3 Available from Aldrich.

4 Tsuda, T.; Satomi, H.; Hayashi, T.; Saegusa, T. J. Org. Chem; 1987, 52, 439-443.

5 Reissig, H.-U.; Angert, H.; Kunz, T.; Janowitz, A.; Handke, G.; Bruce-Adjei, E. J.Org.Chem. 1993, 58, 62806285 .

6 Le Borgne, J.-L. J. Organomet. Chem., 1976, 122, 129-137.

7 Goldstein, S.W.; Overmann, L. E.; Rabinowitz, M. H. J. Org. Chem., 1992, 57, 1179-1190.

8 Goering, H. L.; Tseng, C. C. J. Org. Chem. 1981, 46, 5250-2. 


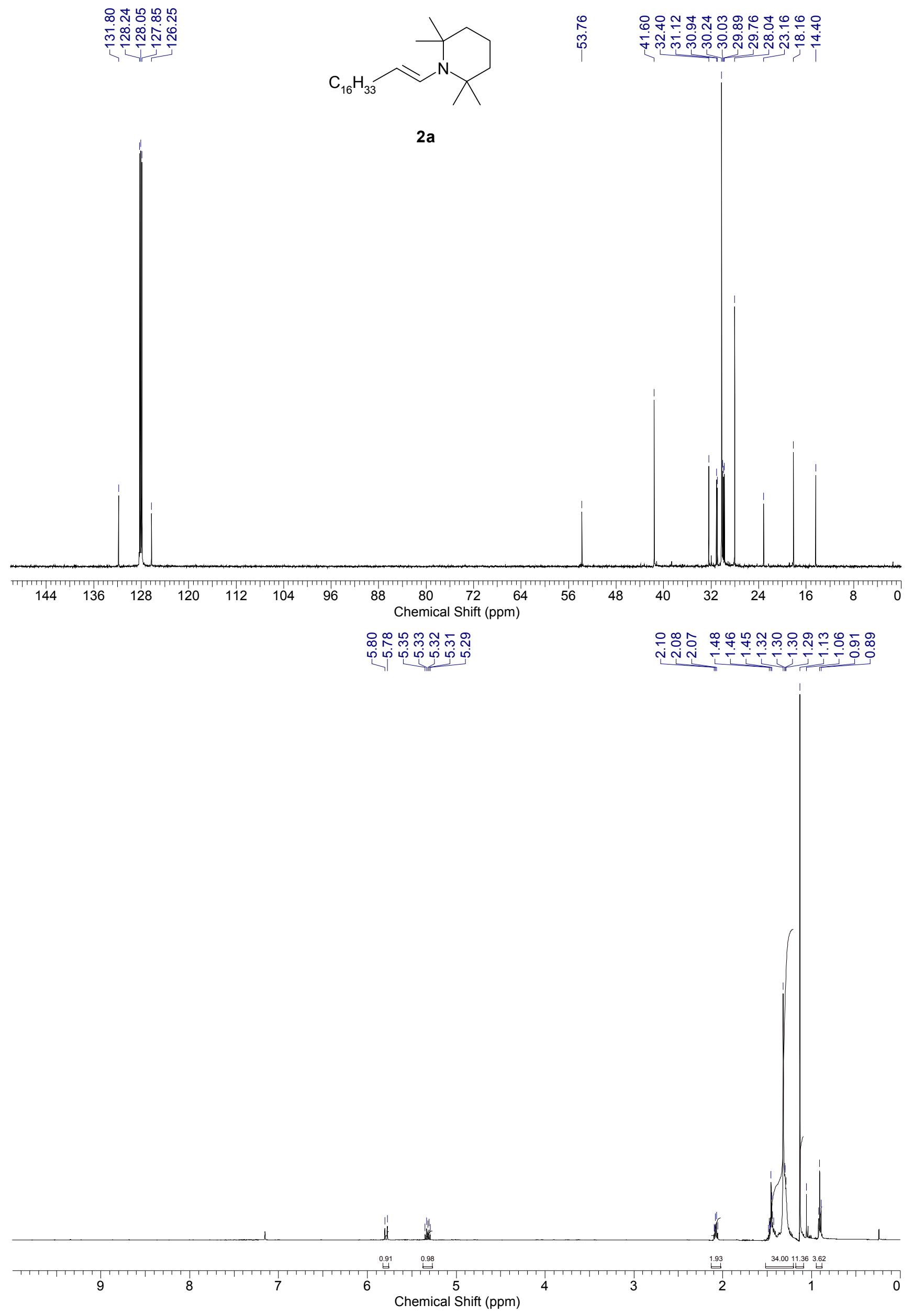


S15

느 웅 용으

్ㅣㅁำ

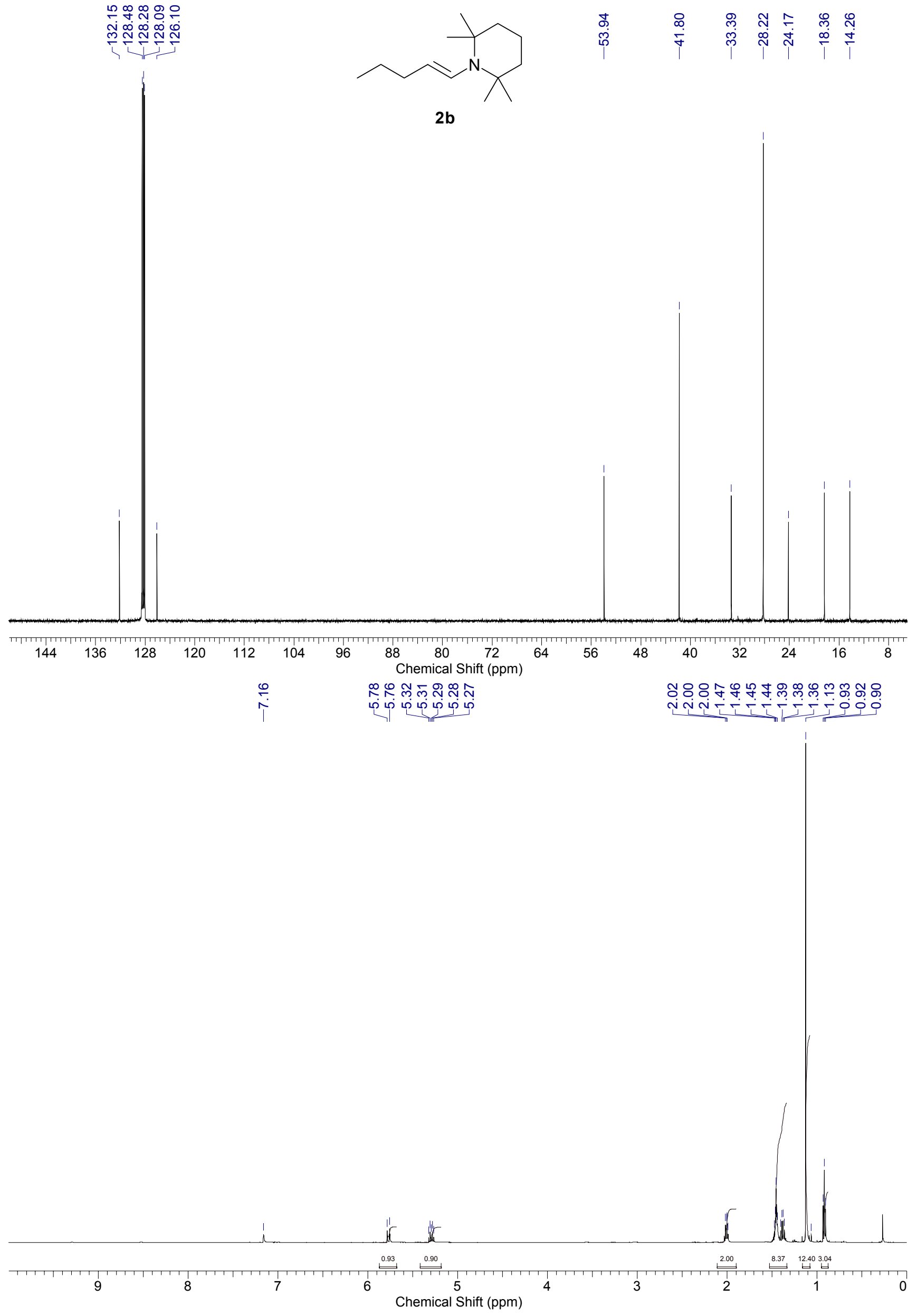




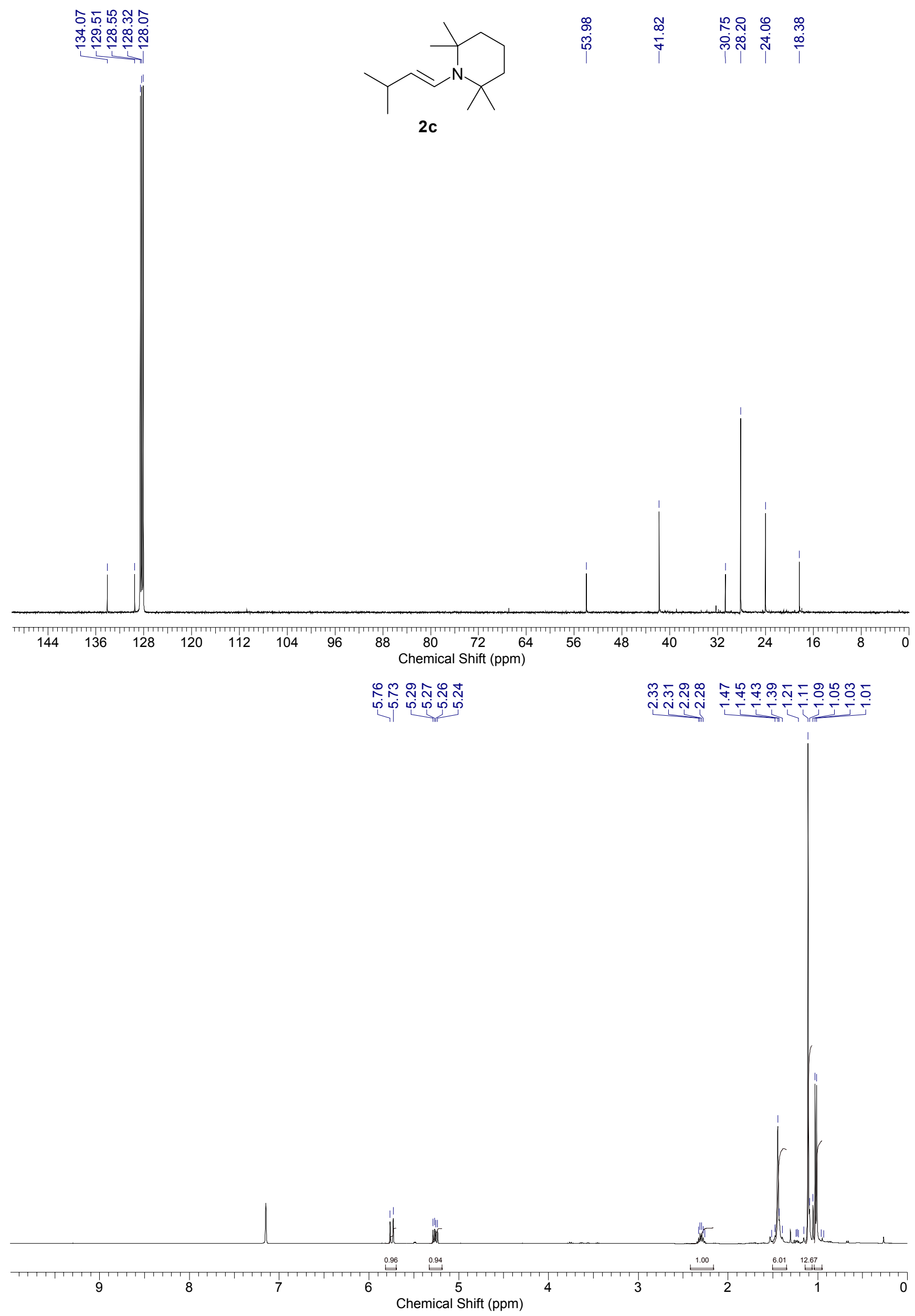




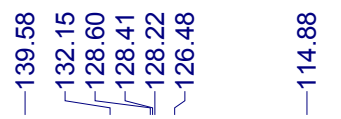

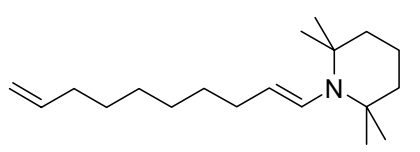

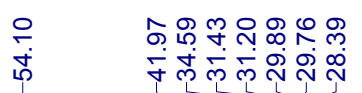

$\underset{\substack{\infty \\ \infty}}{\infty}$

2d

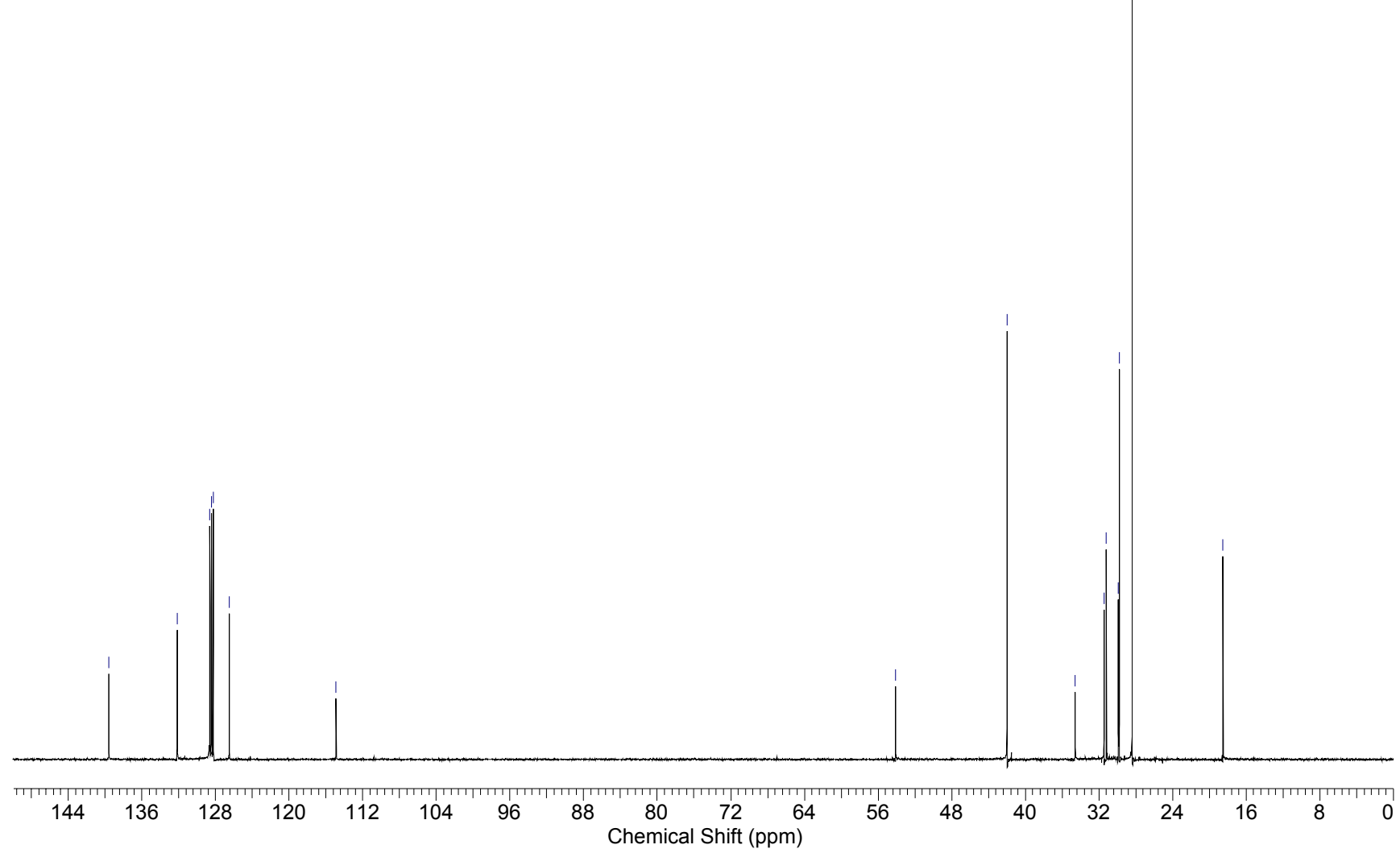

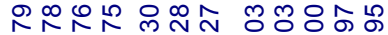
ம்

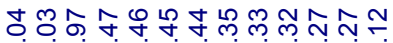

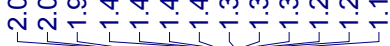

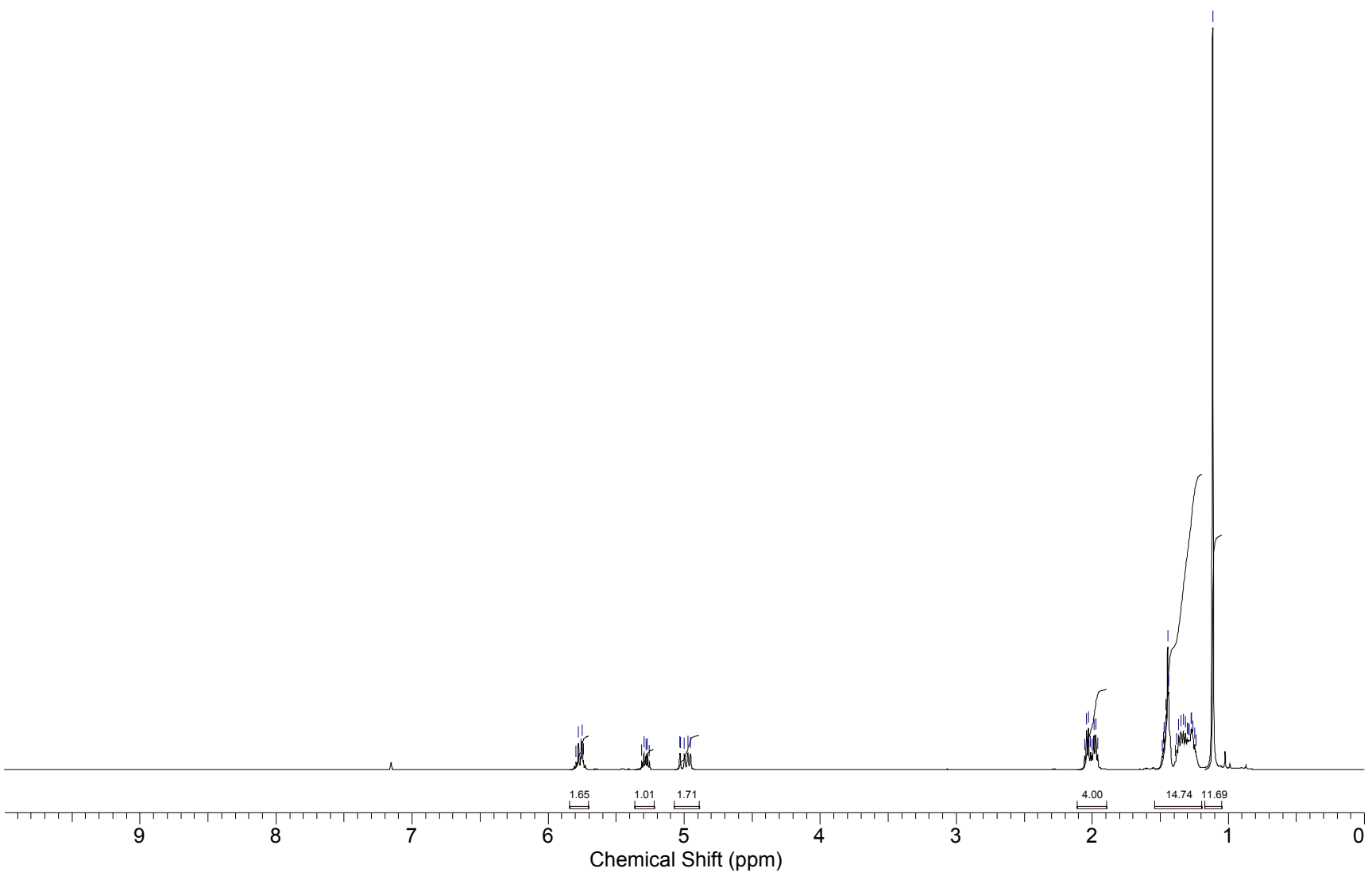




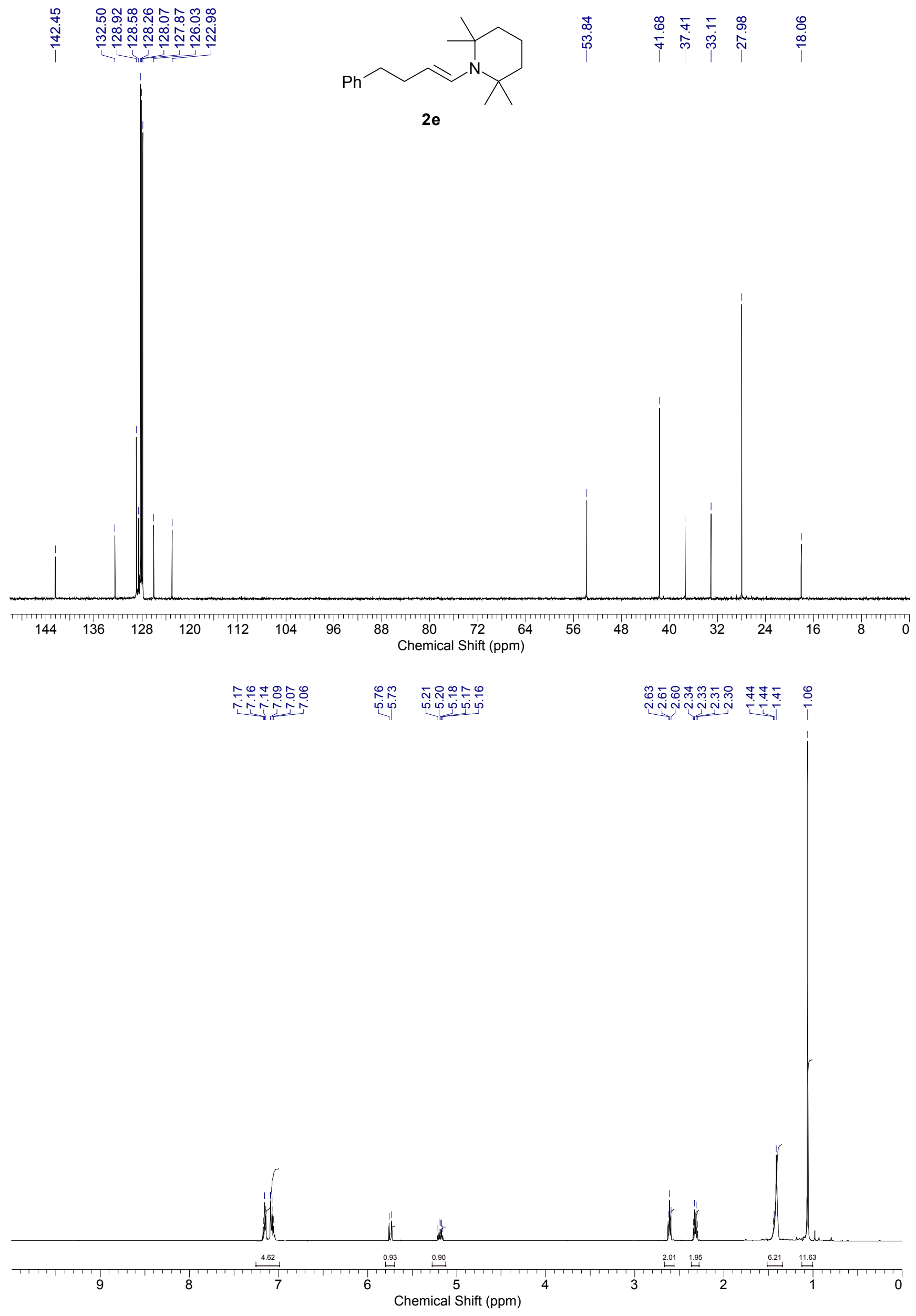



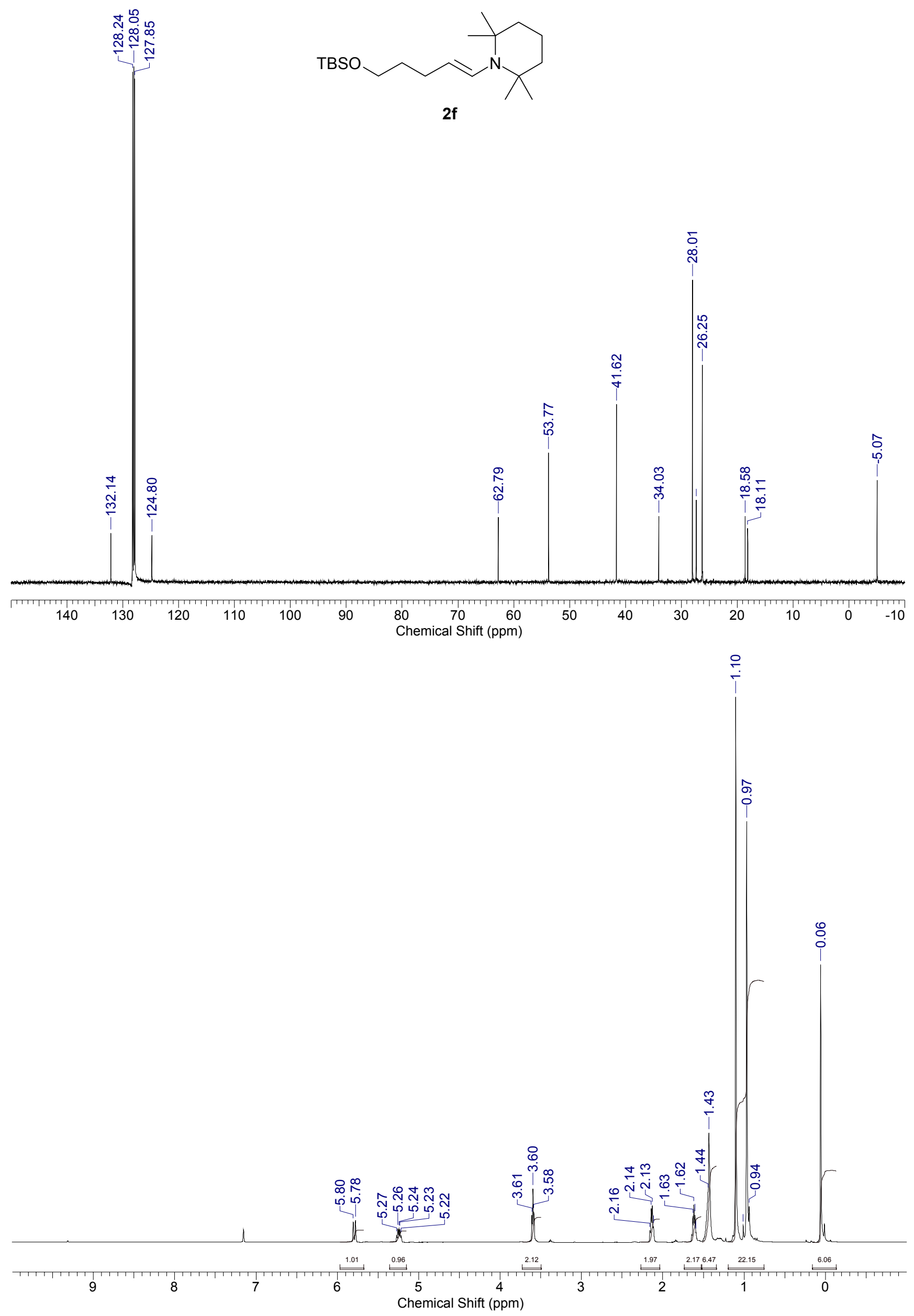


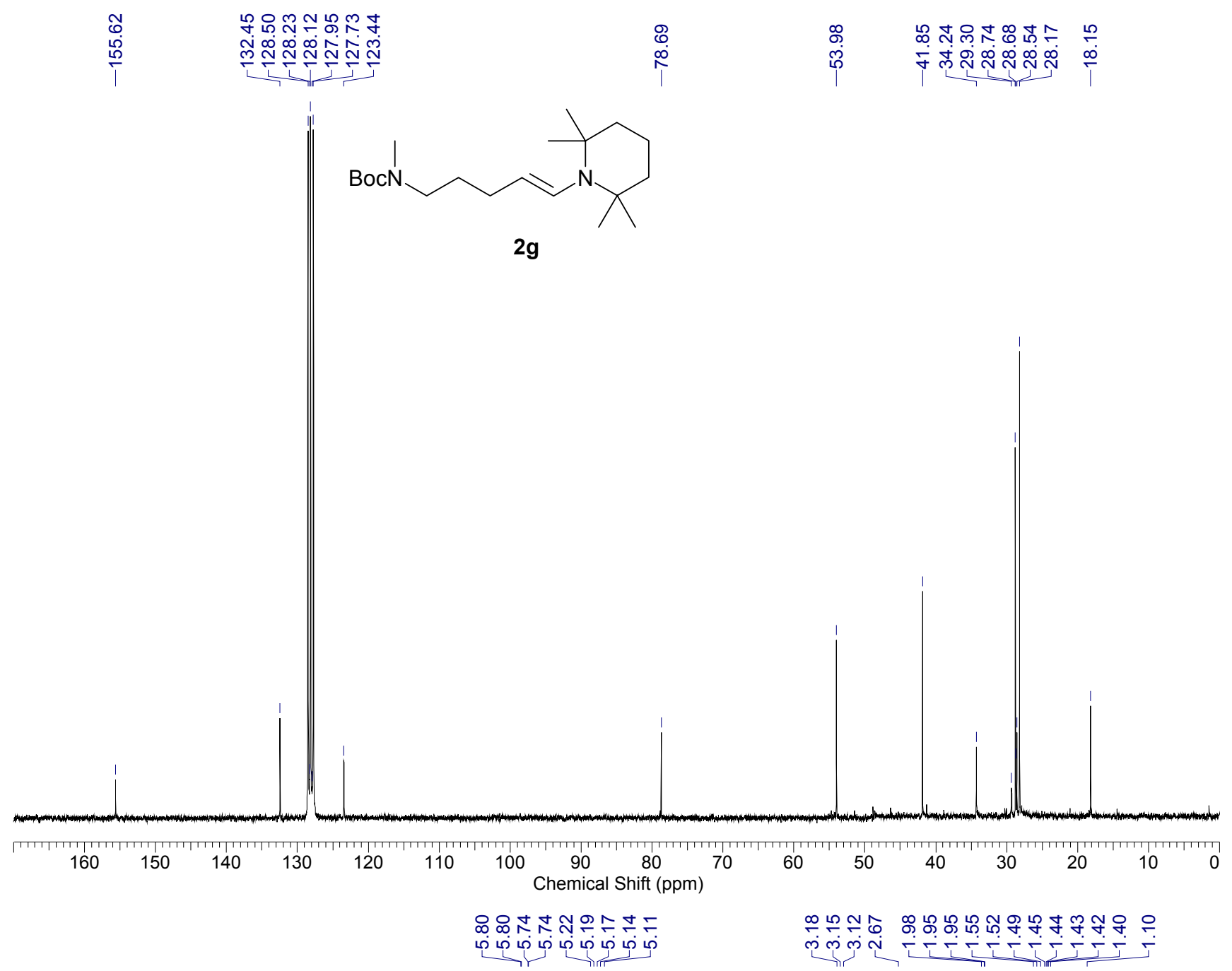

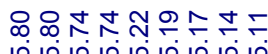

य लm m

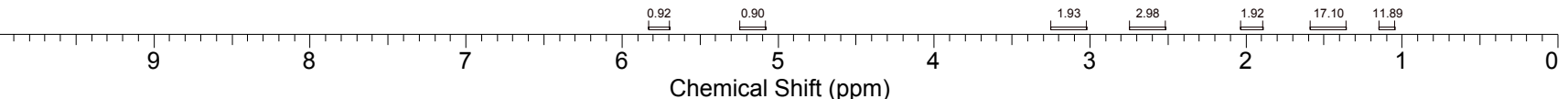




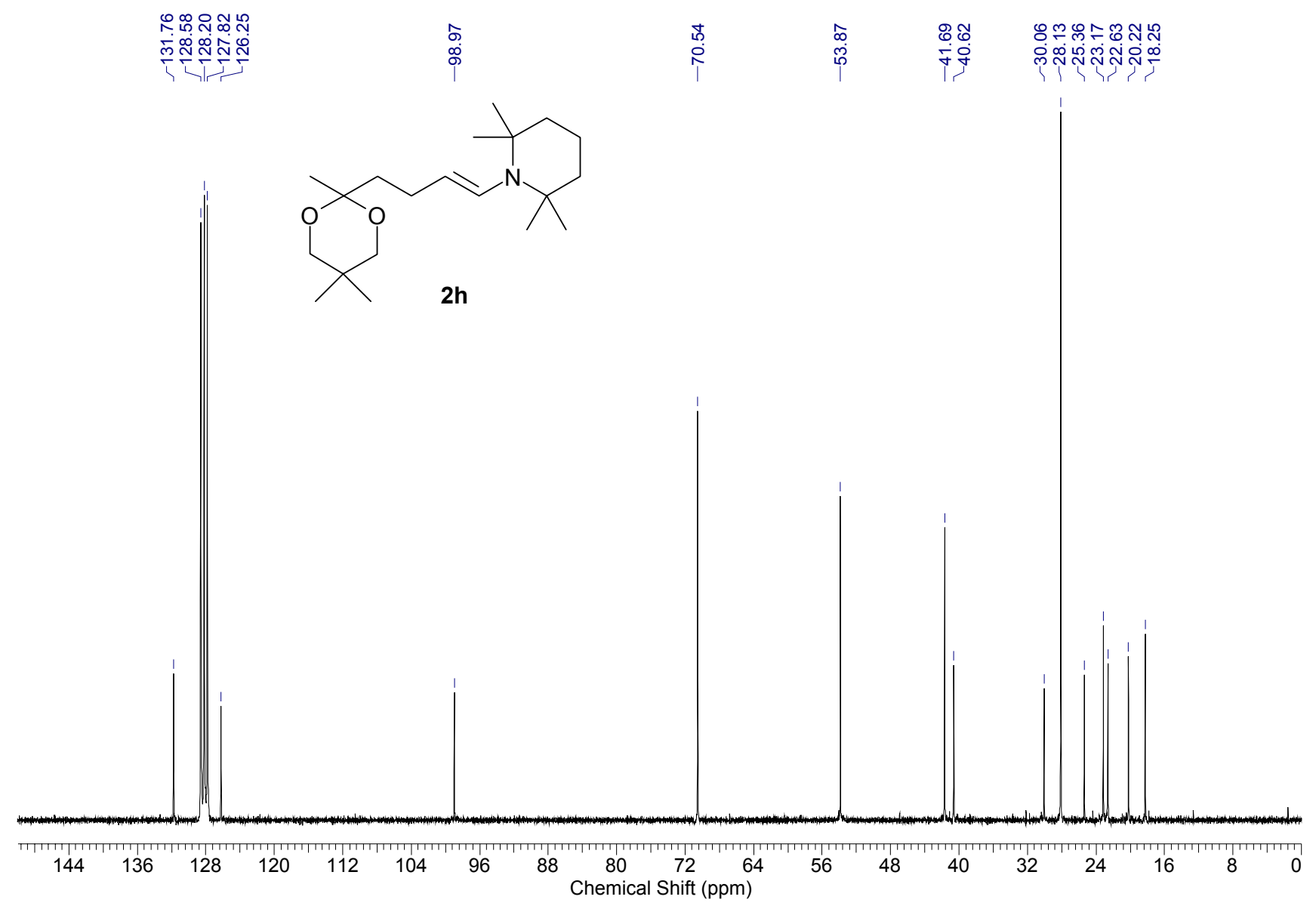

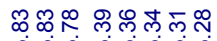

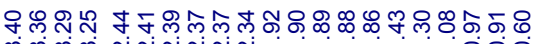

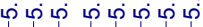

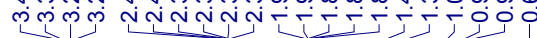

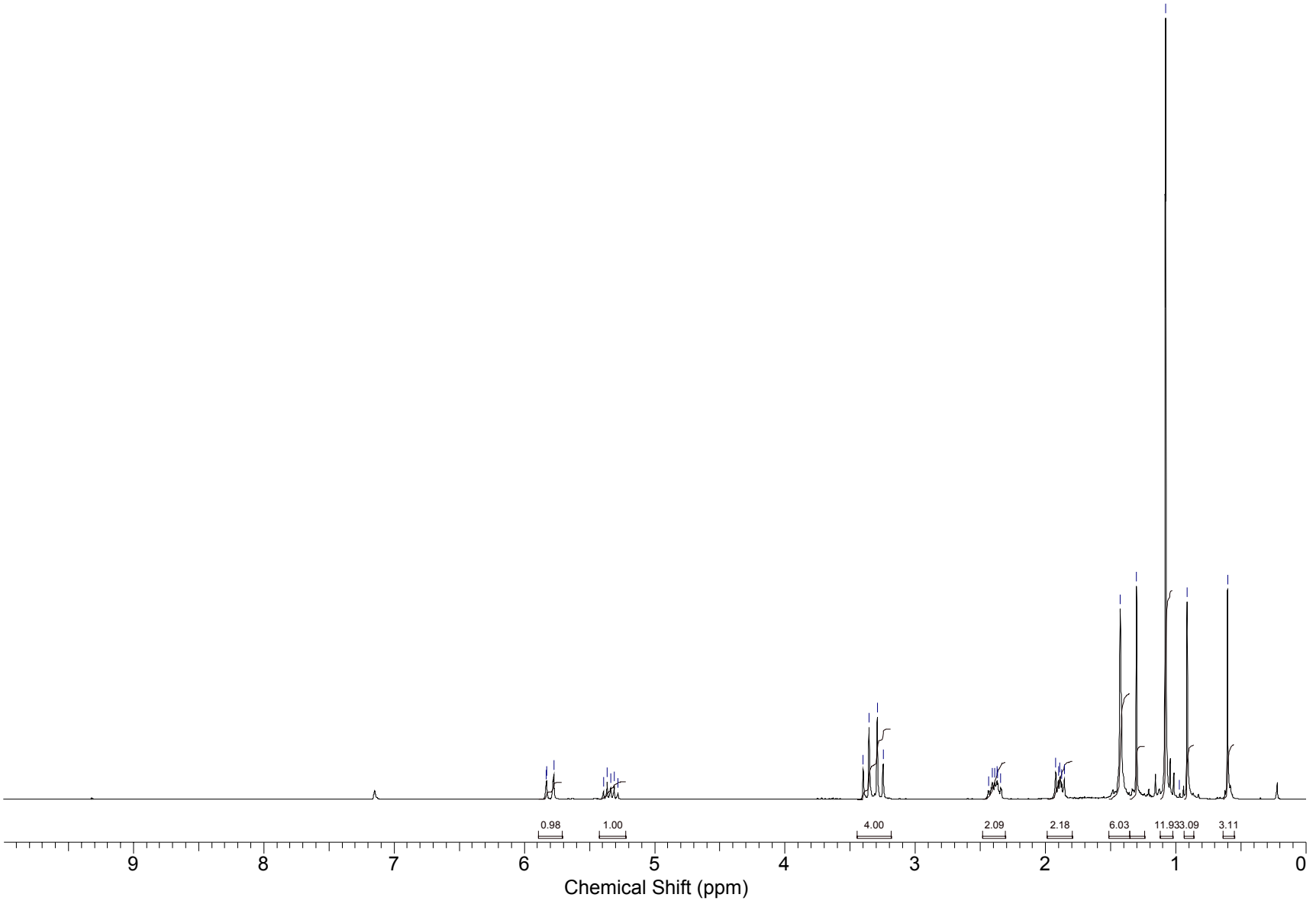




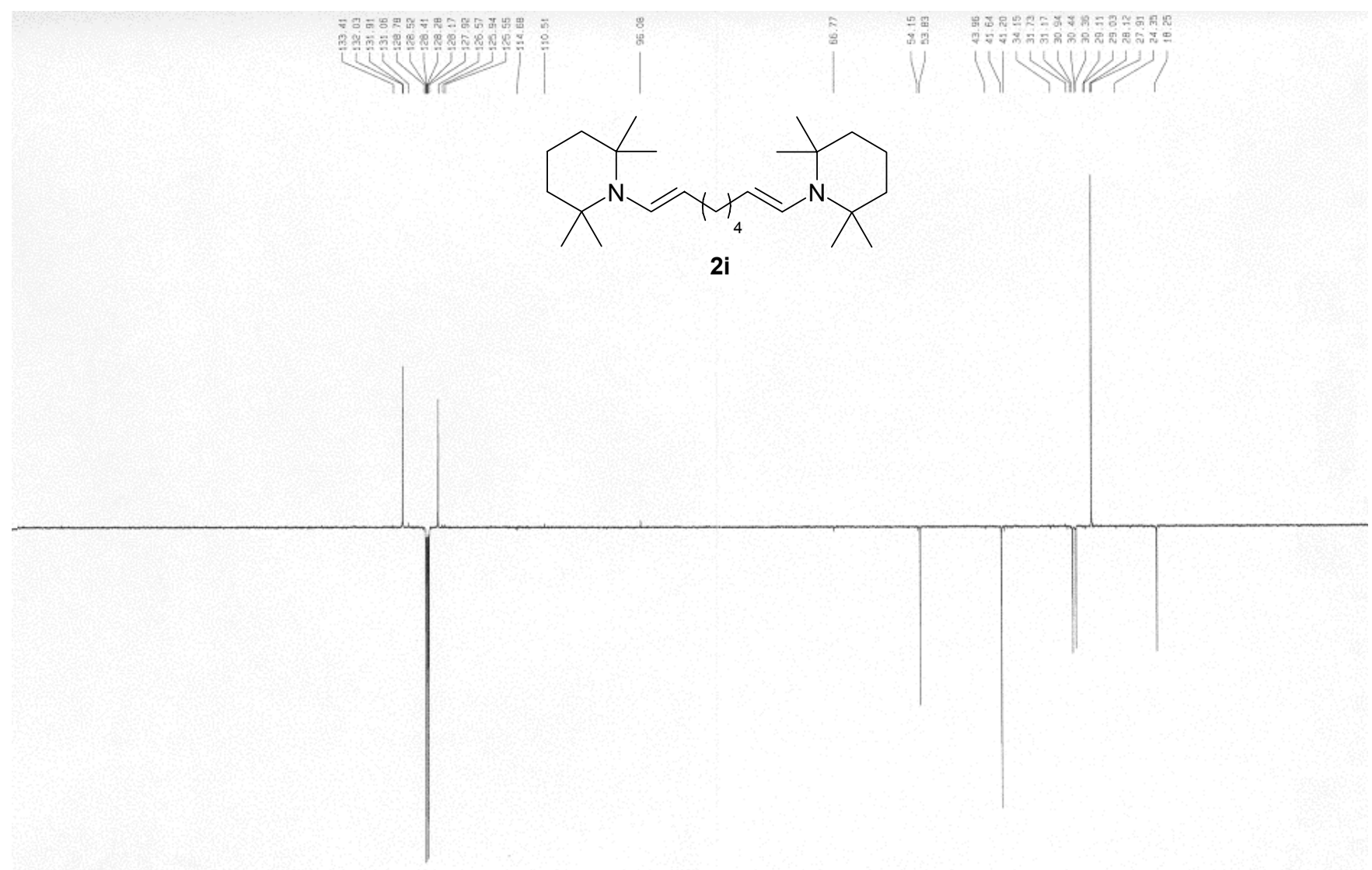

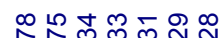

เ่

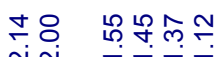

งก

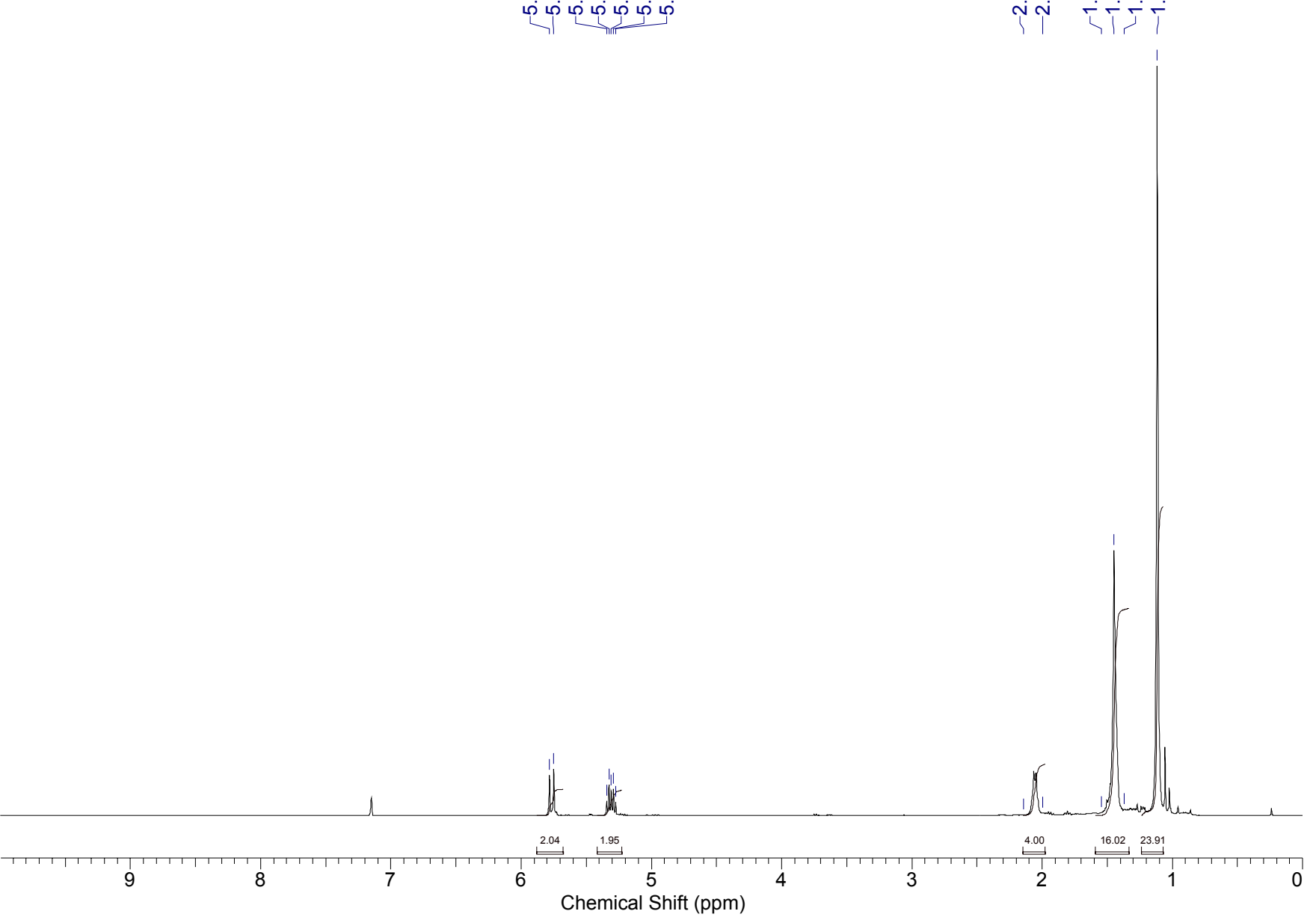



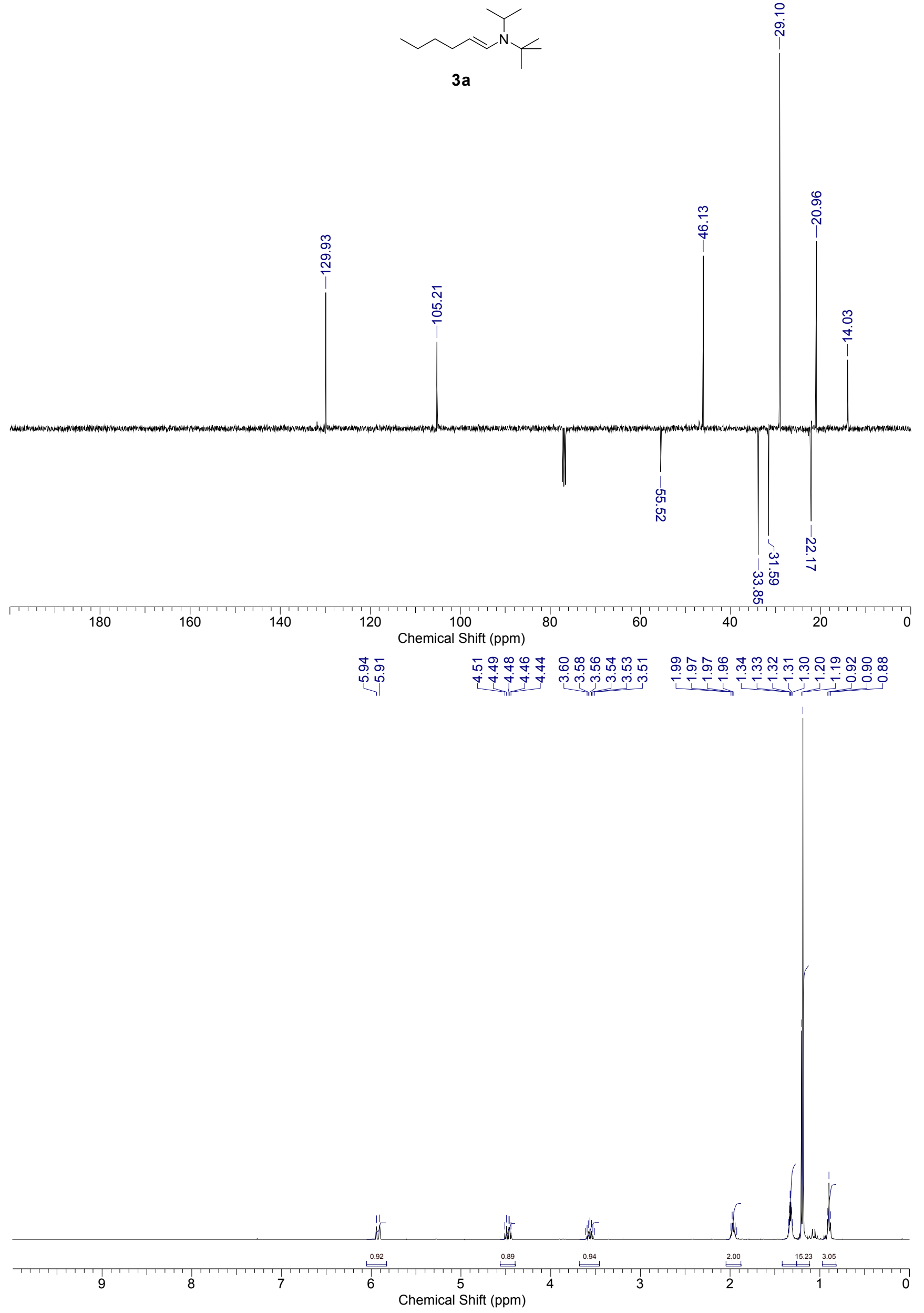


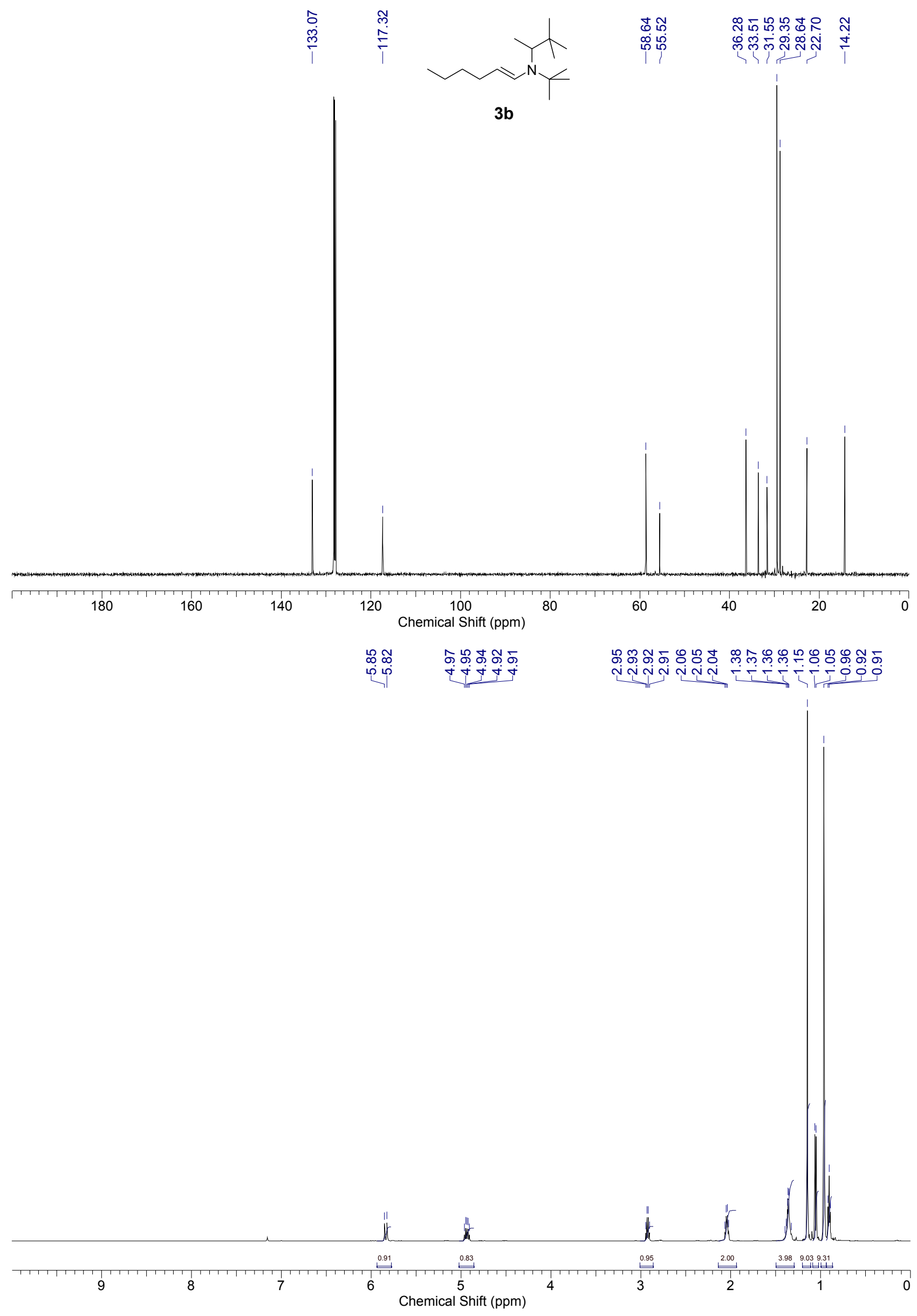


ฆั<smiles>CCCCC(C=O)CCC#N</smiles>

$4 e$

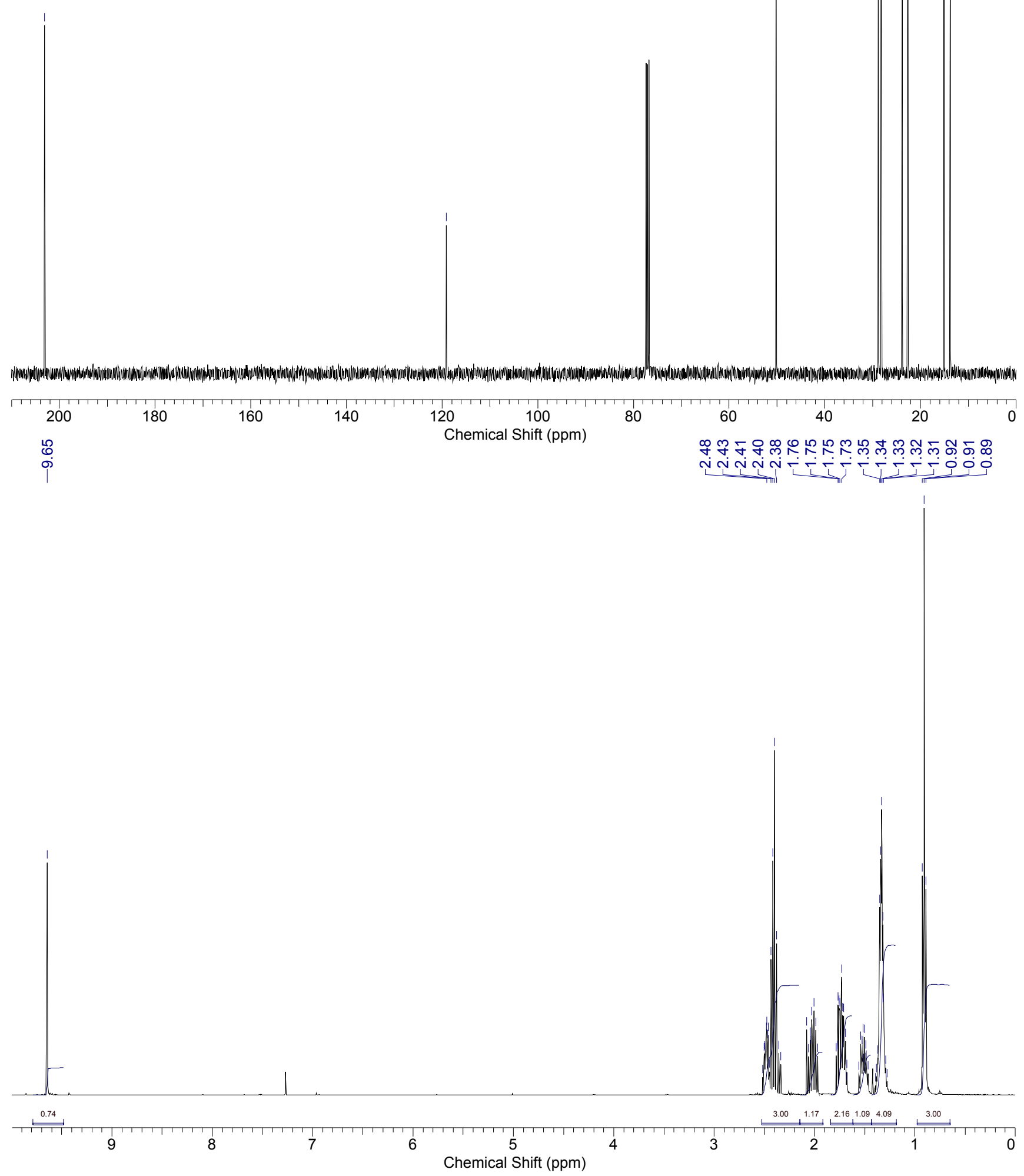



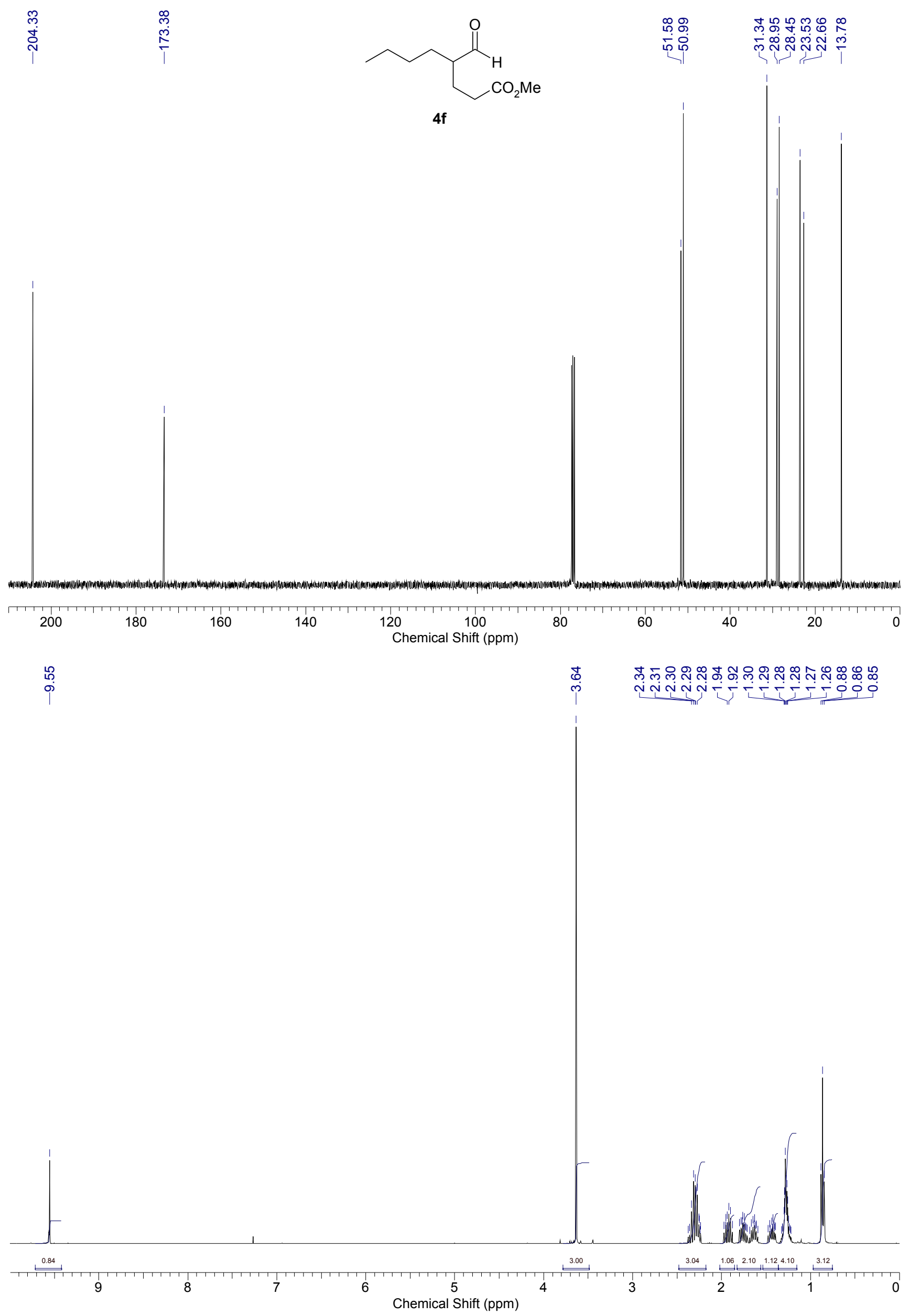
<smiles>CCCCC(C=O)CCCC</smiles>

$4 j$

\section{j}

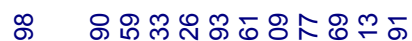

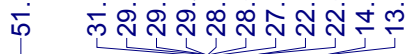

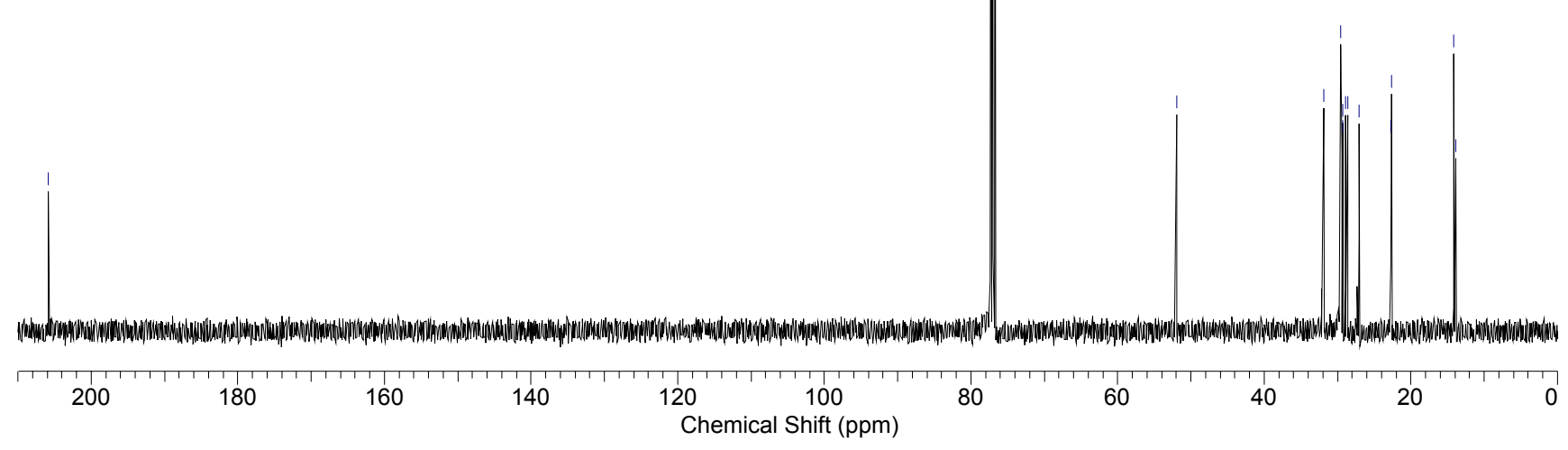

눈 늉

is

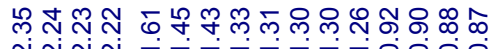

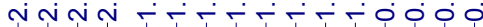

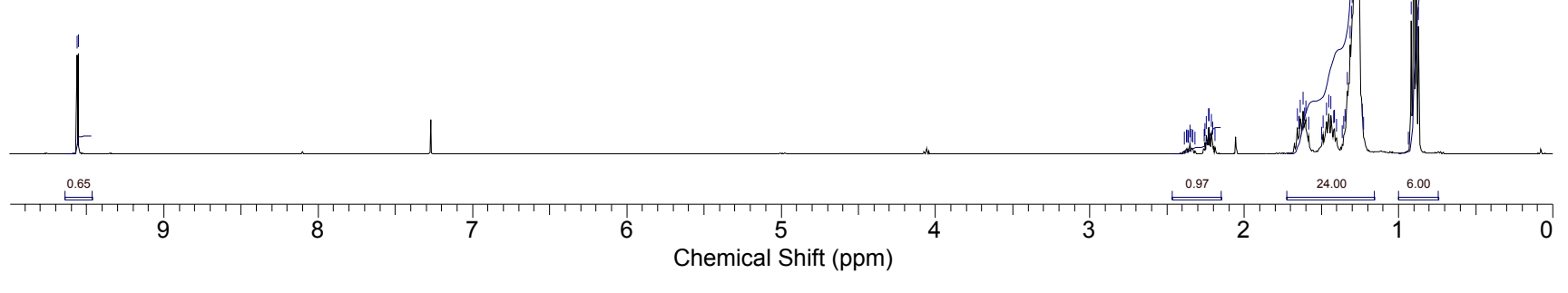


${ }^{7} \mathrm{Li}$ NMR of the reaction mixture when 1,2-epoxypentane was added to $n$-BuLi (1.95 equiv. THF, $25^{\circ} \mathrm{C}$, 1h) (above), and when 1,2-epoxypentane was added to LTMP (1.95 equiv. THF, $25^{\circ} \mathrm{C}, 1 \mathrm{~h}$ ) (below)

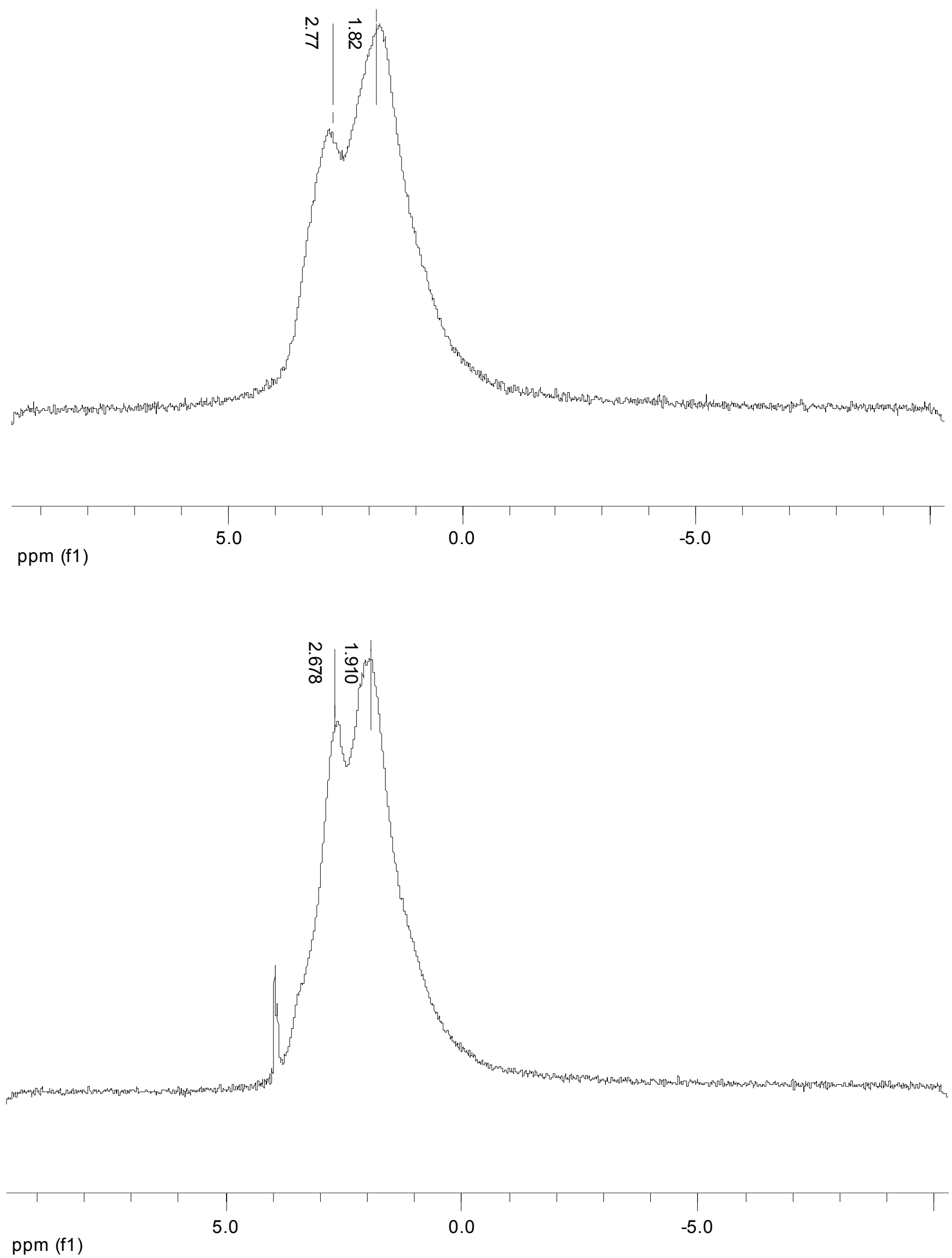

